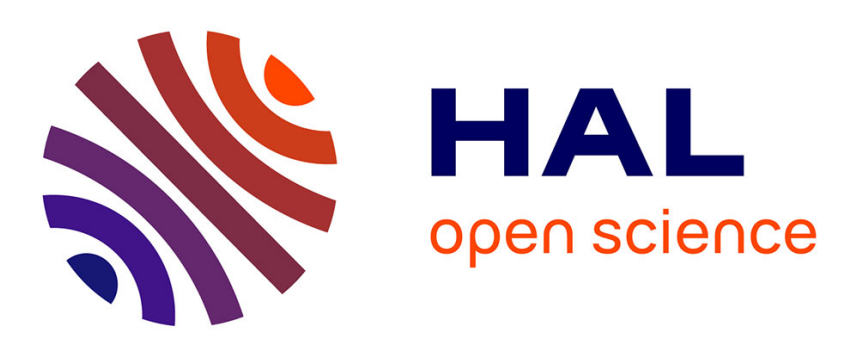

\title{
Optimum Post-Disruption Restoration under Uncertainty for Enhancing Critical Infrastructure Resilience
}

Yiping Fang, Giovanni Sansavini

\section{- To cite this version:}

Yiping Fang, Giovanni Sansavini. Optimum Post-Disruption Restoration under Uncertainty for Enhancing Critical Infrastructure Resilience. Reliability Engineering and System Safety, 2018, 10.1016/j.ress.2018.12.002 . hal-01957113

\section{HAL Id: hal-01957113 \\ https://hal.science/hal-01957113}

Submitted on 17 Dec 2018

HAL is a multi-disciplinary open access archive for the deposit and dissemination of scientific research documents, whether they are published or not. The documents may come from teaching and research institutions in France or abroad, or from public or private research centers.
L'archive ouverte pluridisciplinaire HAL, est destinée au dépôt et à la diffusion de documents scientifiques de niveau recherche, publiés ou non, émanant des établissements d'enseignement et de recherche français ou étrangers, des laboratoires publics ou privés. 


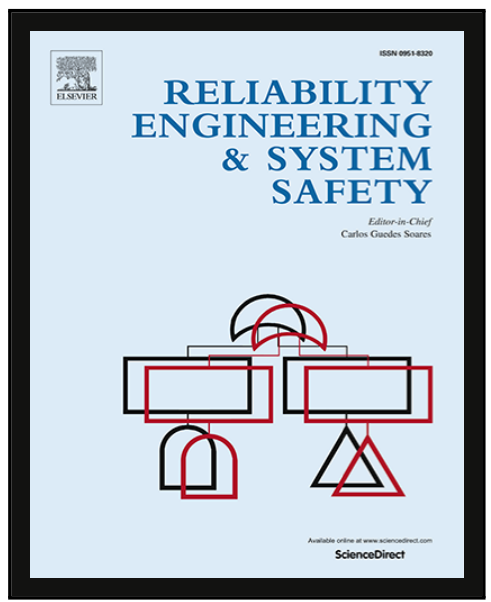

Optimum Post-Disruption Restoration under Uncertainty for Enhancing Critical Infrastructure Resilience

Yi-Ping Fang, Giovanni Sansavini

PII: S0951-8320(17)31006-2

DOI: https://doi.org/10.1016/j.ress.2018.12.002

Reference: RESS 6323

To appear in:

Reliability Engineering and System Safety

Received date:

28 August 2017

Revised date:

30 March 2018

Accepted date:

13 December 2018

Please cite this article as: Yi-Ping Fang, Giovanni Sansavini, Optimum Post-Disruption Restoration under Uncertainty for Enhancing Critical Infrastructure Resilience, Reliability Engineering and System Safety (2018), doi: https://doi.org/10.1016/j.ress.2018.12.002

This is a PDF file of an unedited manuscript that has been accepted for publication. As a service to our customers we are providing this early version of the manuscript. The manuscript will undergo copyediting, typesetting, and review of the resulting proof before it is published in its final form. Please note that during the production process errors may be discovered which could affect the content, and all legal disclaimers that apply to the journal pertain. 


\section{Highlights}

- Propose a stochastic programming for infrastructure restoration under uncertainty

- A multi-mode component repair model of higher practicality is considered

- Propose a tailored Benders decomposition to effectively solve the model

- Show the added value of the stochastic model against its deterministic counterpart

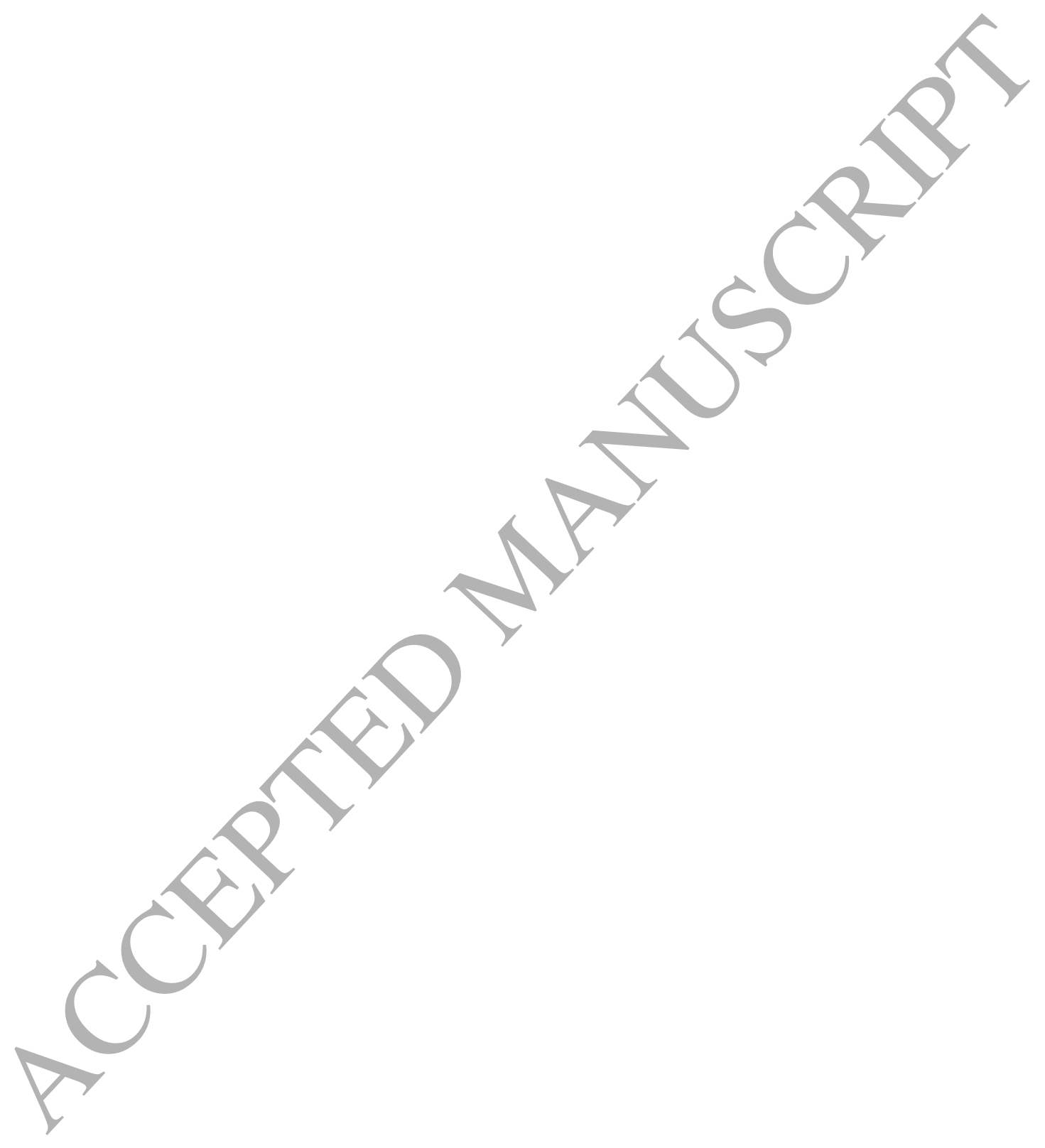




\title{
Optimum Post-Disruption Restoration under Uncertainty for Enhancing Critical Infrastructure Resilience
}

\author{
Yi-Ping Fang ${ }^{1}$ and Giovanni Sansavini ${ }^{2 *}$
}

${ }^{1}$ Chaire Systems Science and the Energy Challenge, Fondation Electricité de France (EDF), Laboratoire Génie Industriel, CentraleSupélec, Université Paris-Saclay, 3 Rue Joliot Curie, 91190 Gifsur-Yvette, France (Email: yiping.fang@centralesupelec.fr)

${ }^{2}$ Laboratory of Reliability and Risk Engineering, Institute of Energy Technology, ETH Zurich, Leonhardstrasse 21, 8092 Zurich, Switzerland (Email: sansavig@ethz.ch)

\section{AbSTRaCT}

The planning of post-disruption restoration in critical infrastructures often relies on deterministic assumptions such as complete information on resources and known duration of the repair tasks. In fact, the uncertainties faced by restoration activities, e.g. stemming from subjective estimates of resources and costs, are rarely considered. Thus, the solutions, obtained by a deterministic approach may be suboptimal or even infeasible under specific realizations of the uncertainties. To bridge this gap, this paper investigates the effects of uncertain repair time and resources on the post-disruption restoration of a critical infrastructure. A two-stage stochastic optimization provides insights for prioritizing the intensity and time allocation of the repair activities with the objective of maximizing system resilience. The inherent stochasticity is represented using a set of scenarios capturing specific realizations of repair activity durations and available resources, and their probabilities. A multi-mode restoration model is proposed that offers more flexibility than its single-mode counterpart. The restoration framework is applied to the reduced British electric power system, and the results demonstrate the added value of using the stochastic model as opposed to the deterministic model. Particularly, the benefits of the proposed stochastic method increase as the uncertainty associated with the restoration process grows. Finally, decision-making under uncertainty largely impacts the optimum repair modes and schedule. 
Keywords: Critical infrastructures; system resilience; restoration planning; uncertainty; stochastic programming.

\section{INTRODUCTION}

Critical infrastructures (CIs) such as power grids, telecommunication networks, transportation networks, are the backbone of our society which depends on their seamless operations to provide services and flows of electrical power, information, energy, materials, etc. [1-4] At the same time, disruptions such as malicious attacks, natural hazards, or technical accidents, become inevitable in today's increasingly complex and risky operating environment [5]. They can significantly impact CIs performance and cause severe economic losses, such as power outages experienced by 2.5 million customers during the 1994 Northridge earthquake in Los Angeles and by 50 million customers during the 2003 North America blackout. Justifiably, then, criticalinfrastructure protection (CIP) has become a priority for all nations $[\underline{2}, \underline{6-8}]$.

In recent years, lessons learned from catastrophic accidents and the acknowledgment of unknown hazards have drawn the focus of CIP studies towards the concept of resilience. In this regard, a system should not only be reliable, i.e. having an acceptably low failure probability, but also resilient, i.e. being capable of effectively absorbing, adapting to and rapidly recovering from disruptive events [르, 9-13]. While resilience can be characterized by different system features and attributes [14], it can be effectively enhanced by developing optimum plans for timely restoring the disrupted service after the occurrence of disruptive events. In this context, the main decision is to determine a schedule of tasks for recovering the failed components $[\underline{15}, \underline{16}]$.

In planning the $\mathrm{CI}$ restoration, resources are often limited during the post-disruption phase, e.g. during the restoration of transportation networks, repair crews and equipment are usually extremely scarce in the immediate aftermath of an earthquake. Hence, optimization approaches are typically used to facilitate the identification and scheduling of effective restoration strategies for the rapid reestablishment of system functionality while accounting for limited amount of resources. Various studies have been proposed in the literature in the context of post-disruption CI restoration into a 
mathematical programming framework. Bryson et al. [17] applied a mixed integer programming (MIP) approach for selecting a set of recovery subplans giving the greatest benefit to business operation. Casari and Wilkie [18] discussed restoration when multiple infrastructures, operated by different firms, are involved. Lee et al. [19] focused on a case of network restoration that involves selecting the location of temporary arcs (e.g., shunts) needed to completely reestablish network services over a set of interdependent networks. A MIP model was proposed to minimize the operating costs involved in temporary emergency restoration. Matisziw et al. [20] proposed an MIP model to restore networks where the connectivity between pairs of nodes is the driving performance metric associated with the network. Nurre et al. [21] studied an integrated network design and scheduling problem for the restoration of CI systems. The problem was formulated using integer programming and a dispatch rule-based heuristics is proposed for its efficient solution. They recently provided a comparative study focusing mainly on model complexity and heuristic dispatch rules for this problem [22]. In addition, González et al. [23] proposed a MIP model for optimal infrastructure system restoration considering joint restoration due to the geographical interdependence between multiple CI systems. Ouyang and Wang [24] studied and compared the effectiveness of five strategies for joint restoration of interdependent infrastructures. Here, they applied a Genetic Algorithm (GA) to generate recovery sequences.

The studies above are based on deterministic assumptions such as complete information on the restoration resources and full knowledge of the activity duration. On the other hand, the restoration of infrastructure systems is complicated by the many decisions to be made in a highly uncertain environment exacerbated by the disaster itself, people's reaction, and limited capability of information gathering. Several factors introduce uncertainty into the parameters of a disaster situation, i.e. available restoration resources and repair crews, the time duration of the repair activity of failed components and the required resources that should be allocated to it. For example, in post-earthquake power systems restoration, all on-duty personnel is likely to be immediately available in the aftermath of the event, but off-duty personnel may have difficulty reporting if the roads are damaged or they have suffered personal injury. Neglecting these uncertainties may lead to budget and schedule overruns, compromised performance and ineffective resource allocation in the system restoration 
process [25]. Therefore, preparations and recovery plans must be robust with respect to many scenarios, for which the modeling of uncertain parameters is significant [26].

To the best of our knowledge, few studies have tackled uncertainty in post-disruption CI restoration.

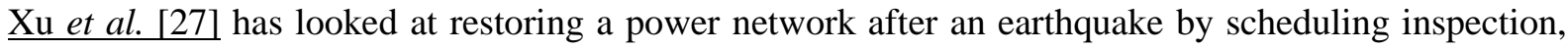
assessment, and repair operations, where the duration of these tasks are assumed to be random variables with known probability distributions. Instead of solving the stochastic program model, the authors used a GA to produce a priority list of repair tasks, which might be suboptimal. Extensive research efforts have been dedicated from the Operation Research (OR) community to the so-called resource-constrained project scheduling problem (RCPSP) under uncertainty [28, 29]. However, existing approaches from the classical RCPSP under uncertainty cannot be directly applied to the CI restoration planning problem (CIRPP) studied in the present paper due to the following challenges: i) there is a precedence network in RCPSP that defines the finish-start precedence relations among the project activities, whereas the relations are not given and thus should be determined in CIRPP; ii) RCPSP is generally based on the minimization of the makespan, i.e. the time to complete the project, whose calculation is trivial, while the evaluation of the objective function in the CIRPP is complicated by system performance quantification through a physical model, e.g. the maximal network flow model [21].

To bridge this research gap, this paper proposes a stochastic programming $[\underline{30}, \underline{31}]$ approach for the post-disruption $\mathrm{CI}$ restoration planning problem (CIRPP) which maximizes the expected system resilience over possible realizations of the uncertain restoration parameters. Specifically, the repair tasks of failed gomponents are modeled as multi-mode activities, in which the repairs are performed with variable allocations of resources, and the processing duration is a function of the amount of allocated resources. For example, the total work content of 18 man-hours can be performed by 1 man in 18 hours, 2 men in 9 hours, or by 1.5 men in 12 hours. Multi-mode repair modeling offers more flexibility than its single-mode counterparts $[\underline{21}, \underline{23}, \underline{27}]$, and is thus of higher practicality for operators of CI systems. To tackle the computational burden of the stochastic program, a tailored Benders decomposition algorithm is proposed to solve the mixed-integer equivalence of the original model, 
where the mixed-integer equivalent problem is obtained by scenario generation (sampling) and reduction techniques [32].

The post-disruption CI restoration planning problem (CIRPP) studied in this paper is related to classical maintenance repair problems (MRPs) [33-37]. However, those two problems are essentially different in terms of the following aspects: I) The CIRPP focuses only on the restoration stage after a single, large-scale disruption on critical infrastructure systems, i.e., assuming the damages to the system components have occurred. Conversely, MRPs usually cover the whole failure and repair process considering component failures as the main source of uncertainties; II) the CIRPP focuses on the identification and scheduling of effective restoration strategies for the rapid reestablishment of system functionality while accounting for limited amount of repair resources. Conversely, MRPs usually focus on the available strategies for system maintenance and repair, e.g., different choices of the maintenance periods for the system components and of the number of repair teams to keep on site [35]; III) the CIRPP is considered as a planning problem and thus studied in a mathematical programming and optimization framework in the literature. Conversely, MRPs typically represent the failure and repair processes from a statistical point of view, describe the processes through stochastic models, e.g., using failure and recovery rates, and adopt simulation methods like Monte-Carlo [ $\underline{34}, \underline{35}]$.

The main contributions of the present study lie in the following aspects: i) a two-stage stochastic programming model is proposed for the post-disruption CIRPP for system resilience, which, to the best knowledge of the authors, is the first effort in the literature to investigate the effects of uncertainty on the post-disruption CI system restoration by OR methods; ii) the modeling of the multi-mode repair of failed components offers more flexibility than its single-mode counterparts, and is thus of higher practicality for operators of CI systems; iii) a tailored Benders decomposition algorithm is proposed to effectively solve the mixed-integer equivalence of the proposed stochastic program model.

The remainder of this paper is organized as follows. Section 2 introduces the multi-mode CI restoration planning problem. Section 3 formulates it as a two-stage stochastic programming model. In Section 4, the proposed solution methodology is presented. Section 5 illustrates the numerical results 
on a test network based on the British electric power transmission system. Finally, concluding remarks and directions for future research are given in Section 6.

\section{Post-Disaster Restoration Problem}

In this study, CI systems are modeled as networks, in which the movement of commodities corresponds to flows and the service is provided if the flows exceed the desired supply level. The CI network is defined as a collection of nodes and links; commodities are exchanged across nodes along paths in the network. Network flow problems have been extensively studied in the literature and fundamentals can be found in [38]. Mathematically, an infrastructure is represented as an undirected graph $\mathbb{G}(V, L)$ comprising a set of nodes (vertices) $V$ connected by a set of links (arcs) $L$. The network nodes are classified into supply nodes $V_{G}$, transshipment nodes $V_{T}$, and demand nodes $V_{D}\left(V_{G} \cup V_{T} \cup\right.$ $\left.V_{D}=V\right)$. Each $\operatorname{arc} l \in L$ is characterized by flow capacity $p_{l}^{\max } \in R_{0}^{+}$, and each supply node $n \in V_{G}$ has a supply capacity $\bar{g}_{n t} \in R_{0}^{+}$in the time period $t$; each demand node $n \in V_{D}$ has a demand $\bar{p}_{n t} \in$ $R_{0}^{+}$in time period $t$ describing nominal operations. System performance, i.e. system service level $F(t)$, is measured as a function of the percentage of demand that can be met throughout the system at time $t$.

$$
F(t)=\frac{\sum_{n \in V_{D}}\left(\bar{p}_{n t}-\Delta p_{n t}\right)}{\sum_{n \in V_{D}} \bar{p}_{n t}}
$$

where $\Delta p_{n t}$ is the amount of unsatisfied demand at node $n \in V_{D}$ at time $t$, and we have $0 \leq \Delta p_{n t} \leq$ $\bar{p}_{n t}$.

The main system components, i.e. supply units, transmission lines, and relay nodes, may be subject to damage stemming from natural disasters or manmade malicious attacks. The proposed restoration planning model/aims to reestablish connectivity between supply and demand nodes in a disrupted area by repairing damaged components over a fixed planning horizon, so that the interruption of services at the demand nodes is minimized and system resilience is maximized. Disruptions are modeled by the removal of a subset of arcs, $L^{\prime} \subset L$, and a subset of nodes $V^{\prime} \subset V$ from the network. The arcs and nodes in sets $L^{\prime}$ and $V^{\prime}$ become non-operational immediately after the disruption, and system performance deteriorates to the minimum level. Successively, system restoration is planned and initiated for ensuring system service by scheduling the individual repair tasks. The planning horizon 
for restoration is divided into discrete time periods, i.e., $t=1 \ldots T$, and $t=0$ identifies the planning instant. The evolution of system performance versus time, i.e. the restoration curve, typically quantifies the level of system resilience following disruptions $[\underline{10}, \underline{14}, \underline{24}]$. Resilience can be measured by the cumulative system service that is restored during the restoration horizon, normalized by the expected cumulative system functionality supposing that the system has not been affected by disruption during this time period $[\underline{10}, \underline{39}]$ :

$$
R(T)=\frac{\sum_{t=1}^{T}[F(t)-F(0)]}{\sum_{t=1}^{T}[T F(t)-F(0)]}, T \geq 1
$$

where $T F(t)$ denotes the targeted system performance if not affected by the disruption, and it is assumed to remain invariant in this study for simplicity. Duration $T$ is defined as the timespan necessary to restore the system functionality to the same level as the original system. The restoration horizon evolves in discrete time periods; therefore, Eq. (2) contains the summation operator. Note that $R(T)$ is in the range of $[0,1]$ since $T F(t) \geq F(t)$ always hold during the restoration process. Then, the system resilience loss is defined as $\Delta R(T)=1-R(T)$.

The restoration of a damaged component $i, \forall i \in L^{\prime} \cup V^{\prime}$, can be performed by variable allocations of resources $h_{i}$, and consequently the repair duration, i.e. the time to repair, $u_{i}$ is a function of such allocation, i.e. $u_{i}=\mathcal{F}\left(h_{i}\right)$. As exemplified in Figure 1, the repair duration is measured in man-hour units defined as the amount of work performed by the average worker during one hour. The higher the number of crews allocated per hour, the shorter repair time for the damaged component, and $u_{i}$ shows a saturation point at which further allocating repair crews does not expedite the repair process. Moreover, there is a minimum amount of resources required for initiating the repair process of a damaged component. The relationship $u_{i}=\mathcal{F}\left(h_{i}\right)$ can vary across different components. In practice, this information can be obtained from the historical repair data of the considered CI system. 


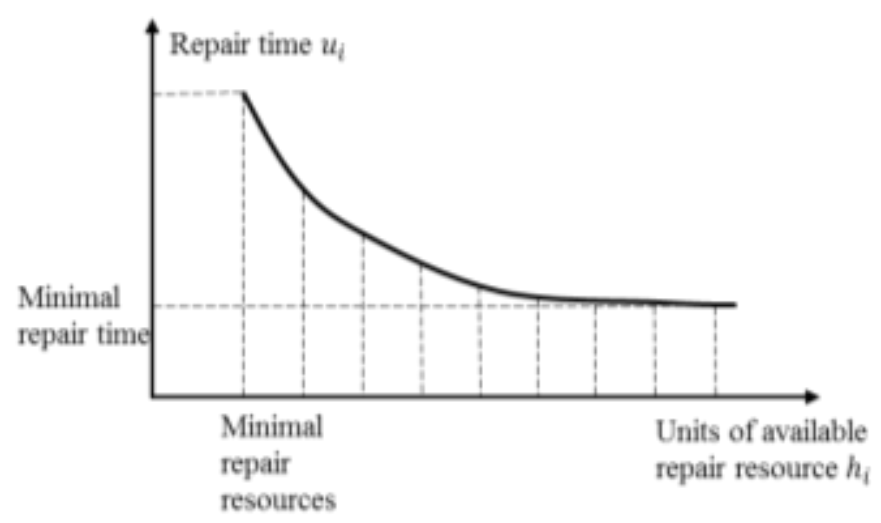

Figure 1. Repair time as a function of the number of allocated repair resources per hour.

In this study, the multi-modal restoration of the damaged component $i$ is represented by pairs of repair duration and allocated resources, i.e. $\left(\tilde{u}_{i}^{m}, h_{i}^{m}\right), m=1 \ldots M_{i} \forall i \in\{n, l\}$, for each discrete repair mode $m$, where $M_{i}$ is the number of modes in which the repair task of $i$ can be implemented. System operators tend to allocate more resources, e.g. repair crews and equipment, to accelerate the restoration of those components which are deemed as critical for the recovery of system functionality. Multiple alternatives usually exist for executing the repair activity based on the combination of the repair modes to which resources are allocated.

The repair time $\tilde{u}_{i}^{m}$ for the repair mode $m=1 \ldots M_{i}$ of component $i$ is a stochastic quantity and is modeled by a random variable that may follow diverse probability distributions. Following the most common choice for the probability distribution of activity time [40], the time to repair is Weibull distributed:

$$
f_{\widetilde{v}_{i}}\left(u ; \alpha_{i}, \beta_{i}\right)=\left\{\begin{array}{cr}
\frac{\alpha_{i}}{\beta_{i}}\left(\frac{u}{\beta_{i}}\right)^{\alpha_{i}-1} e^{-\left(u / \beta_{i}\right)^{\alpha_{i}}}, \text { if } u & \geq 0 \\
0, & u<0
\end{array}\right.
$$

where $\alpha_{i}$ is the shape parameter and $\beta_{i}$ is the scale parameter, $\forall i \in L^{\prime} \cup V^{\prime}$. For the multi-modal repair activity $\left(\tilde{u}_{i}^{m}, h_{i}^{m}\right)$, the assignment of external repair resources to a damaged component leads to a reduction of the expected repair time of the component [25]. Hence, the mean time to repair (MTTR) of component $i$ can be expressed as a function of the allocated resources $h_{i}^{m}$ :

$$
\operatorname{MTTR}_{i}^{m}=\beta_{i}^{m} \Gamma\left(1+\alpha_{i}^{-1}\right)=\mathbb{F}\left(h_{i}^{m}\right)
$$

where $\Gamma$ is the gamma function and $\beta_{i}^{m} \Gamma\left(1+\alpha_{i}{ }^{-1}\right)$ represents the mean of a Weibull random variable, and $\mathbb{F}(\cdot)$ can be any non-increasing function of $h_{i}^{m}$ which account for the decreasing repair efficiency 
as more resources are allocated to the repair task. In this study, $\mathbb{F}(h)=\left(u_{i}^{\max }-u_{i}^{\min }\right) e^{-\gamma h_{i}^{m}}+u_{i}^{\min }$, where $u_{i}^{\max }$ and $u_{i}^{\min }$ are the upper and lower bounds of the expected time to repair for component $i$ and $\gamma$ is the saturation parameter, reflecting the fact that each extra unit of repair resources allocated to mode $m$ has a smaller effect than the previous one due to the decreasing efficiency of recovery activities when the concentration of forces grows. Without loss of generality, any other appropriate relationship can be used to model the saturation of the repair efficiency. Classic parameter estimation techniques such as maximum likelihood and bayesian methods can be applied for calibrating the parameters of the Weibull distribution for the component recovery times, i.e., Eq. (3), and of the relation function, i.e., Eq. (4), based on the historical statistics of the component recovery process..

The total amount of repair resource units that can be allocated to the restoration activities at each time period $t$ is denoted by $\widetilde{H}_{t}, t \in T$, which is usually uncertain at the moment of planning the restoration, i.e. at $t=0$. Therefore, the prediction of $\widetilde{H}_{t}$ for $t \geq 1$ should be made by the decision maker at $t=0$ and it is usually based on expert judgement and past experience. In this work, the prediction is provided in terms of the minimum, the most-likely and the maximum values of $\widetilde{H}_{t}$. Based on the aforementioned representation, the triangular probability distribution is a natural and relatively simple description of the uncertainty associated with the subjective estimation made by the decision maker:

$$
f_{\widetilde{H}_{t}}\left(c ; c_{t}^{-}, c_{t}^{0}, c_{t}^{+}\right)=\left\{\begin{array}{l}
\frac{2\left(c-c_{t}^{-}\right)}{\left(c_{t}^{+}-c_{t}^{-}\right)\left(c_{t}^{0}-c_{t}^{-}\right)}, \text {for } c_{t}^{-}<c \leq c_{t}^{0} \\
\frac{2\left(c_{t}^{+}-c\right)}{\left(c_{t}^{+}-c_{t}^{-}\right)\left(c_{t}^{+}-c_{t}^{0}\right)}, \text { for } c_{t}^{0}<c \leq c_{t}^{+}
\end{array}\right.
$$

where $c_{t}^{-}, c_{t}^{0}, c_{t}^{+}$are the minimum, most-likely and maximum values of $\widetilde{H}_{t}$, respectively, which are estimated by system operators.

In summary, for a disrupted CI system $\mathbb{G}\left(V, V^{\prime}, L, L^{\prime}\right)$, given (i) a set of repair execution modes $m=1 \ldots M_{i}$ for each damaged component $i \in L^{\prime} \cup V^{\prime}$, and (ii) a set of uncertainty characterized by activity duration $\tilde{u}_{i}^{m} \sim f_{\widetilde{U}_{i}}\left(u ; \alpha_{i}, \beta_{i}\right)$ and (iii) by total available repair resources $\widetilde{H}_{t} \sim f_{\widetilde{H}_{t}}\left(c ; c_{t}^{-}, c_{t}^{0}, c_{t}^{+}\right)$, the multi-mode CIRPP attempts to determine the optimum combination of repair modes and the associated starting time under each uncertainty realization which minimizes the expected interruption of services at all the demand nodes and maximize the resilience of the system. 
The multi-mode CIRPP involves determining a repair mode for each failed component, independently of uncertainty scenario, and assigning a starting time to each repair activity under each scenario. The decision process in the CIRPP is a stage-wise scheduling of repair activities subject to resources constraints at each time according to selected execution modes. As the (static) mode selection variables are common to all uncertainty scenarios and do not depend on observed values of random parameters $\tilde{u}_{i}^{m}$ and $\widetilde{H}_{t}$, the problem can be viewed as a two-stage stochastic programming with recourse in which the variables related to the repair mode are determined in the first stage and the repair activities are scheduled in the second stage based on the first-stage decisions.

\section{Two-Stage Stochastic Programming Formulation}

The stochastic multi-mode CIRPP model employs the following notation.

\section{A. Indexes, parameters and sets}

$\mathbb{G}(V, L) \quad$ An undirected graph $\mathbb{G}$ comprising a set of nodes $V$ connected by a set of links $L$ representing the original network system before the disruption.

$V_{G}, V_{T}, V_{D}$ The sets of supply, transshipment and demand nodes, respectively, $V_{G} \cup V_{T} \cup V_{D}=V$

$L^{\prime} \quad$ The set of identified damaged links after a disruption, $L^{\prime} \subset L$

$V^{\prime} \quad$ The set of identified damaged nodes after a disruption, $V^{\prime} \subset V$

$T \quad$ The total number of time periods in the restoration planning

$p_{l}^{\max } \quad$ The capacity of line $l \in L$

$\bar{g}_{n t} \quad$ The supply capacity in supply node $n \in V_{G}$ at time $t$

$\bar{p}_{n t} \quad$ The demand at node $n \in V_{D}$ at time $t$

$M_{l} \quad$ The number of mode for repairing damaged line $l \in L^{\prime}$

$M_{n} \quad$ The number of mode for repairing damaged node $n \in V^{\prime}$

$h_{l}^{m} \quad$ The number of repair resources allocated to a damaged line $l \in L^{\prime}$ under mode $m=$ 1.... $M_{l}$ )

$h_{n}^{m}$ The number of repair resources allocated to a damaged node $n \in V^{\prime}$ under mode $m=1 \ldots M_{n}$

$\tilde{u}_{l}^{m} \quad$ The time duration expected to repair a damaged line $l \in L^{\prime}$ under mode $m=1 \ldots M_{l}$

$\widetilde{u}_{n}^{m}$ The time duration expected to repair a failed node $n \in V^{\prime}$ under mode $m=1 \ldots M_{n}$

$\widetilde{H}_{t} \quad$ The total amount of repair resource units that can be allocated to restoration activity at time $t$

$n \quad$ Index of nodes, $n \in V$

$l \quad$ Index of lines, $l \in L$

$i \quad$ Index of nodes and lines, $i \in\{n, l\}$

$m \quad$ Index of repair model

$s \quad$ Index of the uncertainty scenario which is defined as a realization of all the uncertain 
parameters

\section{B. Decision variables}

$x_{i}^{m} \quad$ Whether or not a damaged component $i \in\{n, l\}$ is selected to be repaired under mode $m$

$\varphi_{i t s} \quad$ Whether or not a damaged component $i \in\{n, l\}$ is assigned to repair capacity at time $t$ under scenario $s$

$\lambda_{i t s} \quad$ Whether or not a failed node $i=n \in V^{\prime}$ or a failed arc $i=l \in L^{\prime}$ is being processed by repair crews at time $t$ under scenario $s$

$\delta_{i t s} \quad$ Operational state (functional/failed) of node $i=n \in V$ or arc $i=l \in L$ at time $t$ under scenario $s$

$f_{\text {lts }} \quad$ The amount of flow passing through arc $l \in L$ at time $t$ under scenario $s$

$g_{n t s} \quad$ The amount of generated flow at node $n \in V_{G}$ at time $t$ under scenario $s$

$\Delta p_{n t s} \quad$ The amount of unsatisfied demand at node $n \in V_{D}$ at time $t$ under scenarios

The problem is formulated as a two-stage stochastic programming with recourse. The general formulation of a two-stage stochastic linear program with recourse is [32]:

$$
\begin{gathered}
\min _{\boldsymbol{x}} z=\boldsymbol{c}^{T} \boldsymbol{x}+\mathbb{E}_{\xi}[\mathcal{H}(\boldsymbol{x}, \xi(s))] \\
\text { s.t. } \boldsymbol{A} \boldsymbol{x}=\boldsymbol{b}, \boldsymbol{x} \in X
\end{gathered}
$$

where $\boldsymbol{c}$ is the cost vector, $\boldsymbol{x}=\left[x_{l}^{m}, x_{n}^{m}\right]$ is the first-stage decision variable vector, $\boldsymbol{A}$ is the coefficient matrix of the first stage variables, $\boldsymbol{b}$ is the right hand side vector and $X$ is the domain of variable $\boldsymbol{x}$. The function $\mathcal{H}(\boldsymbol{x}, \xi(s))$ is the second-stage value function (or recourse function) defined as [41]:

$$
\mathcal{H}(\boldsymbol{x}, \boldsymbol{\xi}(s))=\left\{\begin{array}{c}
\min _{\boldsymbol{y}(s)} \boldsymbol{q}(s)^{T} \boldsymbol{y}(s) \\
\boldsymbol{T}(s) \boldsymbol{x}+\boldsymbol{W}(s) \boldsymbol{y}(s)=\boldsymbol{h}(s) \\
\boldsymbol{y}(s) \in Y
\end{array}\right\}, \forall s \in S
$$

where $\boldsymbol{y}(s)=\left[\varphi_{\text {its }}, \lambda_{i t s}, \delta_{i t s}, f_{l t s}, g_{n t s}, \Delta p_{n t s}\right]$ is the second-stage decision variable vector and $Y$ is its domain, $\boldsymbol{q}(s)$ is the recourse penalty coefficient, $\boldsymbol{T}(s)$ is the coefficient matrix of the first stage variables in the second stage problem's constraints (called technology matrix), and $\boldsymbol{W}(s)$ is the coefficient matrix of the second stage variables in the constraints (called recourse matrix); $\xi(s)$ is a realization of random parameters $\left[\tilde{u}_{i}^{m}, \widetilde{H}_{t}\right]$, indexed by $s$, and $S$ is the scenario index set. The secondstage problem in Eq. (7), wherein decisions are made after the uncertainty on $\tilde{u}_{i}^{m}$ and $\widetilde{H}_{t}$ is cleared, is referred to as the recourse problem.

Specifically, the stochastic programming model of the CIRPP with the objective of minimizing the expected system resilience loss, i.e. maximizing resilience, is: 


$$
Z=\min \mathbb{E}_{\xi(s)} \Delta \tilde{R}(T)
$$

s.t.

$$
\begin{aligned}
& \sum_{l \in L \mid O(l)=n} f_{l t s}-\sum_{l \in L \mid R(l)=n} f_{l t s}=g_{n t s}, \forall n \in V_{G}, \forall t, s \\
& \sum_{l \in L \mid O(l)=n} f_{l t s}-\sum_{l \in L \mid R(l)=n} f_{l t s}=0, \forall n \in V_{T}, \forall t, s \\
& \sum_{l \in L \mid R(l)=n} f_{l t s}-\sum_{l \in L \mid O(l)=n} f_{l t s}+\Delta p_{n t s}=\bar{p}_{n t}, \forall n \in V_{D}, \forall t, s \\
& 0 \leq g_{n t s} \leq \delta_{n t s} \bar{g}_{n t}, \forall n \in V_{G}, \forall t, s \\
& \bar{p}_{n t}\left(1-\delta_{n t s}\right) \leq \Delta p_{n t s} \leq \bar{p}_{n t}, \forall n \in V_{D}, \forall t, s \\
& -\delta_{R(l) t s} \delta_{O(l) t s} \delta_{l t s} p_{l}^{\max } \leq f_{l t s} \leq \delta_{R(l) t s} \delta_{O(l) t s} \delta_{l t s} p_{l}^{\max }, \forall l \in L, \forall t, s \\
& \sum_{m=1}^{M_{i}} x_{i}^{m} \leq 1, \forall i \in\left\{n \in V^{\prime}, l \in \mathcal{L}^{\prime}\right\} \\
& \sum_{t=1}^{T} \varphi_{i t s}=\sum_{m=1}^{M_{i}} x_{i}^{m}, \forall i \in\left\{n \in V^{\prime}, l \in L^{\prime}\right\}, \forall s \\
& \lambda_{i t s}+\delta_{i t s}=\sum_{\tau=1}^{t} \varphi_{i \tau s}, \forall i \in\left\{n \in V^{\prime}, l \in L^{\prime}\right\}, \forall t, s \\
& \delta_{i t s} \geq \delta_{i(t-1) s}, \forall i \in\left\{n \in V^{\prime}, l \in L^{\prime}\right\}, \forall t, s \\
& \sum_{t} t \varphi_{i t s}+\sum_{t} \lambda_{i t s} \leq \sum_{t} t\left[\delta_{i t s}-\delta_{i(t-1) s}\right], \forall i \in\left\{n \in V^{\prime}, l \in L^{\prime}\right\}, \forall s \\
& \sum_{t} \lambda_{i t s} \geq \sum_{m=1}^{M_{i}} u_{i s}^{m} x_{i}^{m}, \forall i \in\left\{n \in V^{\prime}, l \in L^{\prime}\right\}, \forall s \\
& \sum_{i \in L^{\prime} \cup V^{\prime}} \lambda_{i t s} \sum_{m=1}^{M_{i}} h_{i}^{m} x_{i}^{m} \leq H_{t s}, \forall t, s \\
& \delta_{i t s}=1, \forall i \in\left\{n \in V \backslash V^{\prime}, l \in L \backslash L^{\prime}\right\}, \forall t, s \\
& x_{i}^{m} \in\{0,1\}, \forall i \in\left\{n \in V^{\prime}, l \in L^{\prime}\right\}, m=1 \ldots M_{i} \\
& \varphi_{i t s}, \lambda_{i t s}, \delta_{i t s} \in\{0,1\}, \forall i \in\left\{n \in V^{\prime}, l \in L^{\prime}\right\}, \forall t, s
\end{aligned}
$$


The system resilience loss under each scenario in the objective function (8) is calculated by Eqs. (1) and (2). Constraints (9)-(14) are the constraints for the operation of general infrastructure network systems, i.e. constraints (9)-(11) guarantee the flow balance at generation, transship and demand nodes, respectively, constraints (12) ensure that the flow generated at a supply node does not exceed its capacity if it is operational, constraints (13) ensure that the amount of flow delivered to a demand node does not exceed the requested demand, and constraints (14) force the arc flows to comply with capacities taking into account that a flow can exist only if the arc and its ending nodes are functional. Furthermore, constraints (15)-(21) capture the relationships among the restoration/scheduling decisions. Constraints (15) ensure that only one repair mode is chosen for each damaged component. Constraints (16) state that if a damaged component is selected to be repaired, then a repair resource must be assigned to it. Constraints (17) enforce that a damaged component assigned to a repair crew is either under repair or functional, i.e. its restoration is completed. Constraints (18) ensure that restored components do not fail again over the planning horizon. Constraints (19) states that components are immediately available as soon as the repair workforce completes the task. Moreover, constraints (20) ensure that once the restoration of a component is started, it continues at least for the estimated time to repair duration for the chosen repair mode. Constraints (21) limit the use of available repair resources at each time unit. Finally, constraints (22)-(24) describe the binary nature of the decision variables.

The above CIRPP model (8)-(24) is most applicable to single-commodity infrastructure systems which include, for instance, power, water/waste water, natural gas, and supply chain systems. It conveniently extended to multi-commodity systems by adding different indexes to the network flow variables

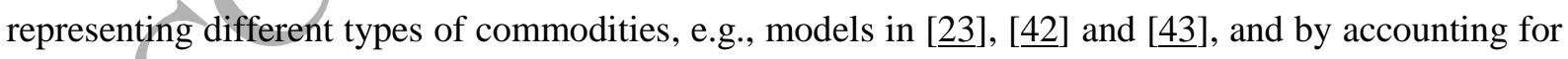
additional constraints of multi-commodity systems such as the interdependency among the flows of different commodities [표.

\section{Solution MethodologY}

\section{A. Scenario Generation and Reduction}

Owing to the presence of continuous random variables, i.e. Weibull-distributed repair times for each damaged component and triangular-distributed repair resources at each time step, the stochastic 
parameter vector of the proposed model $\xi$ has an infinite support. To obtain a solution to the problem, the stochastic parameter vector $\xi$ needs a discrete representation which restricts the possible outcomes to a finite set. In this study, Latin hypercube sampling [44] is applied to replace $\xi$ via a scenario tree approximation which builds a finite and large set of uncertainty scenarios, $\Omega$, over which a discrete probability distribution $Q$ is defined. The Latin hypercube sampling guarantees that the whole range of the random variables is spanned. For a sample size of $N$, the Latin hypercube sampling technique selects $N$ different values of the random variables by dividing their range into $N$ disjoint intervals. Then, $N$ scenarios are built by shuffling and pairing these values; the probability of each scenario is $1 / N$

The computational tractability of the stochastic optimization model is achieved by reducing the number of scenarios while still preserving the essential features of the original sampling set. In other words, we seek a reduced scenario set that yields an optimum solution whose value is close to the solution of the original optimization problem. Consider the uniform discrete probability distribution $Q$ over the sampled scenario set $\Omega$, the scenario reduction problem amounts to determining a scenario subset $\Omega_{r} \subset \Omega$ of cardinality $N_{r}$ and assigning new probabilities $Q^{\prime}$ to the reduced scenarios such that [32]:

$$
\begin{gathered}
D_{k}\left(Q, Q^{\prime}\right)=\inf _{\eta}\left\{\sum_{\substack{s \in \Omega \\
s^{\prime} \in \Omega_{r}}} v\left(s, s^{\prime}\right) \eta\left(s, s^{\prime}\right): \eta\left(s, s^{\prime}\right) \geq 0, \forall s \in \Omega, \forall s^{\prime} \in \Omega_{r}, \sum_{s^{\prime} \in \Omega_{r}} \eta\left(s, s^{\prime}\right)\right. \\
\left.=\pi_{s}, \forall s \in \Omega, \sum_{s \in \Omega} \eta\left(s, s^{\prime}\right)=\varpi_{s^{\prime}}, \forall s^{\prime} \in \Omega_{r}\right\}
\end{gathered}
$$

where $D_{k}\left(Q, Q^{\prime}\right)$ is called Kantorovich distance and is the most common probability distance used in stochastic programming, $\pi_{s}$ and $\varpi_{s^{\prime}}$ represent the probabilities of scenarios $s$ and $s^{\prime}$ in sets $\Omega$ and $\Omega_{r}$ according to probability distributions $Q$ and $Q^{\prime}$, respectively, and $v\left(s, s^{\prime}\right)$ is a nonnegative, continuous, symmetric function, often referred to as cost function and is represented by a norm defined in the scenario space. Further details about Kantorovich distance can be found in [45].

The above problem can be solved through diverse techniques; in this paper, the fast forward selection algorithm is selected because the reduced sets defined by this heuristic algorithm perform well in 
practice $[46,47]$. The development and application of the fast forward selection algorithm are detailed

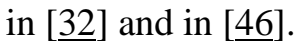

In summary, the infinite support of the stochastic parameter vector of the proposed model $\xi$ is firstly reduced to a sample size of $N$ by Latin hypercube sampling and then to a computationally tractable number of scenarios, $N_{r}\left(N_{r}<N\right)$, using the fast forward selection algorithm. The scenario reduction process implements the fast forward selection method and ensures that the reduced scenario set has the closest probability distance with the original sample space by solving the Kantorovich distance problem (25). Therefore, the reduced scenario set has the theoretical guarantee to be an unbiased representation of the original probability distribution.

\section{B. Tailored Benders Decomposition Strategy}

By sampling the scenario tree and reducing it to a tractable number of scenarios, the proposed stochastic programming is converted to its deterministic mixed-integer programming equivalence. As a result of this, a Benders' decomposition method can be applied to its solution which offers an efficient strategy for large-scale MIP. As opposed to the original problem, the relaxed master problem (RMP) and the Benders' subproblem (SP) in the Benders' decomposition are much more tractable. This section proposes a tailored Benders decomposition algorithm for the effective solution of the CIRPP (8)-(24).

In the classic Benders decomposition framework [48], an original MIP problem is transformed into an RMP and an SP that are iteratively solved and the solution of one problem is given as input to the other, until the optimal solution is achieved. At each iteration, there are two types of cuts that can be generated based on the solution of the SP and then are added to the RMP: i) The first type is optimality cuts that attempt to increase the lower bound of the minimization problem (or the upper bound of the maximization problem), which is obtained from the RMP. ii) The second type of cuts is feasibility cuts, which are used to remove the RMP solutions that are not feasible in the SP. Instead of using only one SP at each iteration, a set of subproblems are formulated and solved, one for each of the $S$ scenarios. Thus, multiple cuts can be generated and added into the RMP at each iteration. showed that, By doing so, the computational performance of the algorithm improves [31]. Given an instance of the binary 
decision vector $\overline{\mathbf{z}}_{s}=\left[\bar{x}_{i}^{m}, \bar{\varphi}_{i t s}, \bar{\lambda}_{i t s}, \bar{\delta}_{i t s}\right]$, the SP associated with problem (8)-(24) under scenario $s$ is formulated as:

$\operatorname{SP}\left(\overline{\mathbf{z}}_{S}\right)$

$$
\min \sum_{t=1}^{T} \sum_{n \in V_{D}} \Delta p_{n t s}
$$

s.t. (9)-(14) for one specific scenario $s$ and $\delta_{i t s}=\bar{\delta}_{i t s}$

Since $\operatorname{SP}\left(\overline{\mathbf{z}}_{S}\right)(26)-(27)$ is a pure linear program, the strong duality theory holds, and its dual problem, called dual subproblem $\operatorname{DSP}\left(\overline{\mathbf{z}}_{S}\right)$, can be compactly written as:

$\operatorname{DSP}\left(\overline{\mathbf{z}}_{S}\right)$

$$
\begin{gathered}
\max \left(\boldsymbol{b}-\boldsymbol{B} \overline{\mathbf{z}}_{S}\right) \boldsymbol{\pi}_{s} \\
\text { s.t. } \boldsymbol{\pi}_{s} \in \boldsymbol{\Pi}
\end{gathered}
$$

where $\boldsymbol{b}$ is the right-hand side vector of (27), $\boldsymbol{B}$ is the left-hand side coefficient matrix of (27), $\boldsymbol{\pi}_{S}$ is the dual variable vector corresponding to constraint (27) and $\Pi$ represents the dual feasible region to $\operatorname{SP}\left(\overline{\mathbf{z}}_{s}\right)$. The set of extreme points of $\boldsymbol{\Pi}$ is denoted as $\operatorname{vert}(\boldsymbol{\Pi})$, and the set of extreme rays of $\boldsymbol{\Pi}$ is denoted as ray( $(\boldsymbol{\Pi})$. In the iterative Benders decomposition, the RMP is formulated as follows: RMP

$$
\min _{z} \sum_{s} P_{s} \eta_{s}
$$

s.t.

$$
\begin{aligned}
& \eta_{s} \geq\left(\boldsymbol{b}-\boldsymbol{B} \boldsymbol{z}_{s}\right) \overline{\boldsymbol{\pi}}_{S}^{k}, \forall k \in \mathcal{O}_{K}, s \in S \\
& 0 \geq\left(\boldsymbol{b}-\boldsymbol{B} \boldsymbol{z}_{S}\right) \overline{\boldsymbol{\pi}}_{S}^{k}, \forall k \in \mathcal{F}_{K}, S \in S
\end{aligned}
$$

(15)-(24)

where $P_{s}$ is the probability associated with scenario $s$ and $\eta_{s}$ is new introduced slack variables; In (31) we have $\overline{\boldsymbol{\pi}}_{s}^{k} \in \operatorname{vert}(\boldsymbol{\Pi})$, for $k \in \mathcal{O}_{K}$, and (31) defines the set of currently available Benders optimality cuts, and it is obtained via solving the $\operatorname{DSP}\left(\overline{\mathbf{z}}_{S}\right)$ for all the scenarios $s \in S$ in the previous iterations; In (32) we have $\overline{\boldsymbol{\pi}}_{s}^{k} \in \operatorname{ray}(\boldsymbol{\Pi})$, for $k \in \mathcal{F}_{K}$, and (32) defines the set of currently available Benders feasibility cut. At a given iteration of the Benders decomposition, RMP is solved first to obtain the optimal solutions of $\overline{\mathbf{z}}_{S}^{k}$. Then, the optimal solutions of $\overline{\mathbf{z}}_{S}^{k}$ are used to solve $\operatorname{DSP}\left(\overline{\mathbf{z}}_{S}^{k}\right)$ and generate the 
optimal solutions (or the extreme ray if $\operatorname{DSP}\left(\overline{\boldsymbol{z}}_{S}^{k}\right)$ is unbounded) of the dual variables $\overline{\boldsymbol{\pi}}_{s}^{k}$ and a new optimality cut in the form of (31) (or feasibility cuts in the form of (32)) to include into the RMP for each scenario $s \in S$. The objective of the RMP provides a lower bound to the optimal solution of the original problem (8)-(24), and the probability-weighted sum of $\operatorname{DSP}\left(\overline{\mathbf{z}}_{S}^{k}\right)$, i.e. $\sum_{s} P_{S}\left(\boldsymbol{b}-\boldsymbol{B} \overline{\mathbf{z}}_{S}^{k}\right) \overline{\boldsymbol{\pi}}_{S}^{k}$, for all the scenarios provides an upper bound.

For the proposed CIRPP model, it can be observed that:

Proposition 1. For a given binary variable vector $\overline{\mathbf{z}}_{s}=\left[\bar{x}_{i}^{m}, \bar{\varphi}_{i t s}, \bar{\lambda}_{i t s}, \bar{\delta}_{i t s}\right]$ which satisfy the constraints (15)-(24), the Benders' primal subproblem $\operatorname{SP}\left(\overline{\mathbf{z}}_{S}\right)$ (26)-(27) is always feasible and bounded, i.e. $\boldsymbol{\Pi} \neq \varnothing$, and the dual subproblem $\operatorname{DSP}\left(\overline{\mathbf{z}}_{S}\right)(28)-(29)$ is always feasible and bounded and it has at least one optimum solution.

Proposition 1 is easily proven by noticing that the solution characterized by all network flow variables $\left(f_{l t s}, g_{n t s}\right)$ equal to 0 and by demand shedding equal to the overall demand $\left(\Delta p_{n t s}=\bar{p}_{n t}\right)$ is always feasible. This property circumvents the need to generate feasibility cuts in the decomposition procedure, therefore only optimality cuts are generated and added to the RMP in each iteration, and the convergence of the algorithm is accelerated.

The detailed procedure of the proposed Benders decomposition procedure encompasses the following steps:

\section{Benders decomposition algorithm}

Step 0. $U B \leftarrow \infty, L B \leftarrow 0$, iteration counter $K=0$

Step 1. Solve the RMP (30)(31)(33) to obtain its optimal solution $\left(\overline{\mathbf{z}}_{s}, \bar{\eta}_{s}\right), L B \leftarrow \sum_{s} P_{S} \bar{\eta}_{S}$ Step 2. For each $s \in S$

Solve the DSP( $\left.\overline{\boldsymbol{z}}_{S}\right)(28)-(29)$, obtain its optimal solution $\overline{\boldsymbol{\pi}}_{S}$ and objective value $\left(\boldsymbol{b}-\boldsymbol{B} \overline{\mathbf{z}}_{S}\right) \overline{\boldsymbol{\pi}}_{S}$

End for $U B \leftarrow \sum_{S} P_{S}\left(\boldsymbol{b}-\boldsymbol{B} \overline{\mathbf{z}}_{S}\right) \overline{\boldsymbol{\pi}}_{S}$

Step 3. If $U B-L B \leq \epsilon$ (where $\epsilon$ is a predefined tolerance) then stop. Otherwise,

(a) Add a total number of $|S|$ Benders optimality cut $\eta_{s} \geq\left(\boldsymbol{b}-\boldsymbol{B} \boldsymbol{z}_{s}\right) \overline{\boldsymbol{\pi}}_{s}^{k}, \forall s \in S$ to the RMP (30)(31)(33) 
(b) $K \leftarrow K+1$

Go to Step 1.

The RMP (30)-(33) is a mixed integer nonlinear programming owing to the products of binary variables, i.e. $\delta_{R(l) t s} \delta_{O(l) t s} \delta_{l t s}$ and $\lambda_{i t s} x_{i}^{m}$, in constraints (14) and (21) of the original CIRPP. According to [49], both nonlinearities can be conveniently replaced by equivalent linear expressions by introducing two sets of binary variables $u_{l t s}$ and $v_{i t s}^{m}$ which satisfy the constraints:

$$
\begin{gathered}
u_{l t s} \leq \delta_{R(l) t s}, u_{l t s} \leq \delta_{O(l) t s}, u_{l t s} \leq \delta_{l t s}, \forall l, t, s \\
u_{l t s} \geq \delta_{R(l) t s}+\delta_{O(l) t s}+\delta_{l t s}-2, \forall l, t, s \\
v_{i t s}^{m} \leq \lambda_{i t s}, v_{i t s}^{m} \leq x_{i}^{m}, \forall i, t, s, m \\
v_{i t s}^{m} \geq \lambda_{i t s}+x_{i}^{m}-1, \forall i, t, s, m .
\end{gathered}
$$

The new binary variables $u_{l t s}$ and $v_{i t s}^{m}$ replace the products of $\delta_{R(l) t s} \delta_{O(l) t s} \delta_{l t s}$ and $\lambda_{i t s} x_{i}^{m}$, respectively, in the original RMP formulation (30)-(33). Therefore, the RMP becomes a mixed-integer linear programming that can be efficiently solved by ayailable off-the-shelf branch-and-cut software, e.g. CPLEX [50].

\section{Case Study}

The proposed CIRPP is exemplified with reference to a test infrastructure network based on the British high-voltage electrical power transmission system $[\underline{51}, \underline{52}]$. The original power system includes $400 \mathrm{kV}$ and $275 \mathrm{kV}$ transmission levels, and the reduced representative network comprises 29 nodes and 99 lines, as shown in Figure 2. The network operation data can be found in [52]. For ease of representation, this analysis considers constant generation capacities and load levels, and only transmission lines can be damaged following a disruption. The number of repair crews is the limited resource allocated to repair damaged lines. Without loss of generality, the shape parameters of the Weibull distribution of the repair time for all components are assumed to be equal to 5, with which the Weibull distribution is generally a symmetric and bell-shaped curve. Each damaged line can be repaired in two modes, i.e. $M_{l}=2, \forall l \in L^{\prime}$, with one or two allocated repair crews, i.e., $h_{l}^{1}=1$ or 2 , and the associated expected repair times are $\beta_{i}^{1} \Gamma\left(1+5^{-1}\right)=10$ and $\beta_{i}^{2} \Gamma\left(1+5^{-1}\right)=7$, respectively. 
As suggested in [53], the MTTR for transmission line restoration is around 10 hours under the assumption of normal repair workforce.

The considered disruption involves three levels of system failures causing direct damage and loss of functionality to $5,10,15$ transmission lines $\left(\left|L^{\prime}\right|=5,10,15\right)$. These levels of damage reflect the magnitude of occurred events, e.g. a power blackout in South Australia on October 27, 2016 was caused by severe wind that brought down 6 high-voltage power lines [54]. All the restoration cases are analyzed in a 32-h restoration planning horizon, which is divided into four 8-hours shifts. The chosen planning horizon ensures that all the failed components can be repaired. The parameters of the triangular distribution of the available repair crews during each shift are given in Table 1. The number of available repair crews and the associated uncertainty in a shift are always larger or equal to the same values during earlier shifts. This assumption takes into account the fact that on-duty repair crews are usually available right after the disruption, while all off-duty resources cannot be available immediately; rather their number increase with time [55]. The available number of repair crews within each shift is assumed to be constant. In order to investigate the benefits of using the stochastic model as opposed to the deterministic model that considers only expected values of activity durations, two problems are considered for each disruption case:

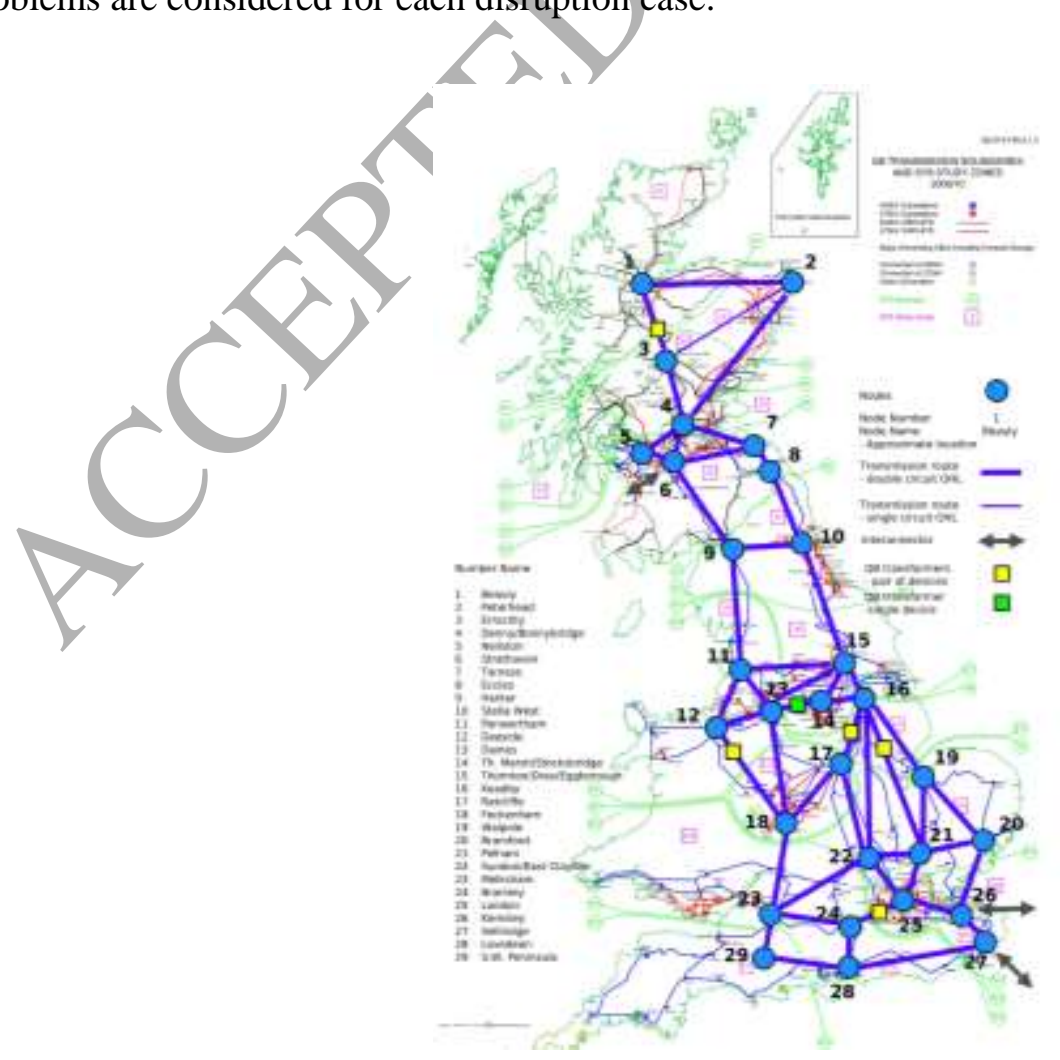


Figure 2. The test network model of the British electric power transmission system.

Table 1. Parameters of the triangular probability distribution of the available repair crews in each shift

\begin{tabular}{cccc}
\hline \hline$i$-th shift & $c_{t}^{-}$ & $c_{t}^{0}$ & $c_{t}^{+}$ \\
1 & 2.0 & 3.0 & 4.0 \\
2 & 4.0 & 5.0 & 6.0 \\
3 & 6.0 & 8.0 & 10.0 \\
4 & 10.0 & 12.0 & 14.0 \\
\hline \hline
\end{tabular}

I) Stochastic program problem (SPP): 1000 independent uncertainty scenarios are generated using the Latin hypercube sampling method [44]. With the use of the fast forward reduction algorithm [32], the number of the uncertainty scenarios is reduced to 5 , then, the corresponding equivalent MIPs are solved.

II) Expected value problem (EVP): the deterministic counterpart of the stochastic program is solved, which considers only the expected values of the repair parameter, i.e. expected activity durations and expected available repair crews in each shift.

The aim of solving these two problems is to quantify the value of the stochastic solution (VSS), defined as the difference between the stochastic solution and the expected value solution [31]. Specifically, let $Z_{S P P}=\min _{x} \mathbb{E}[f(\boldsymbol{x}, \xi)]$ be the optimum solution value of the SPP where $f(\boldsymbol{x}, \boldsymbol{\xi})$ represent the objective $\Delta \widetilde{R}(T)$ in $(8), \boldsymbol{x}_{E V P}=\arg \min _{\boldsymbol{x}} f(\boldsymbol{x}, \mathbb{E}(\xi))$ and $Z_{E V P}=\mathbb{E}_{\xi}\left[f\left(\boldsymbol{x}_{E V P}, \boldsymbol{\xi}\right)\right]$ be the solution and expected solution value of EVP, then, the difference $Z_{E V P}-Z_{S P P}$ is referred to as the value of the stochastic solution (VSS) [56]. Furthermore, in this case study, we simply the objective function (8) to $\Delta \tilde{R}(T)=\sum_{t=1}^{T} \sum_{n \in V_{D}} \Delta p_{n t s}$ by ignoring all the constant items in Eqs. (1) and (2) in order to retain the dimension.

The restoration problems for different disruption cases are solved using the proposed Benders decomposition method, in which the RMP and DSP are solved by CPLEX [50]. Figure 3 shows the objective function values, i.e. the cumulative unsatisfied demand (CUD), for all the six instances, i.e. SPP and EVP under three disruption cases $\left(\left|L^{\prime}\right|=5,10,15\right)$. The VSS is $0.007 \mathrm{GWh}, 0.215 \mathrm{GWh}$ and $0.386 \mathrm{GWh}$, for disruption scenarios $\left|L^{\prime}\right|=5,10,15$, respectively. Given a household consumes 10.4 
$\mathrm{KWh}$ /day electricity in the UK [57], this is equivalent to the daily electricity consumption of around 673, 20'673 and 37'115 household, respectively. This demonstrates an obvious benefit in using the stochastic method over the deterministic one based solely on the expected uncertain parameters.

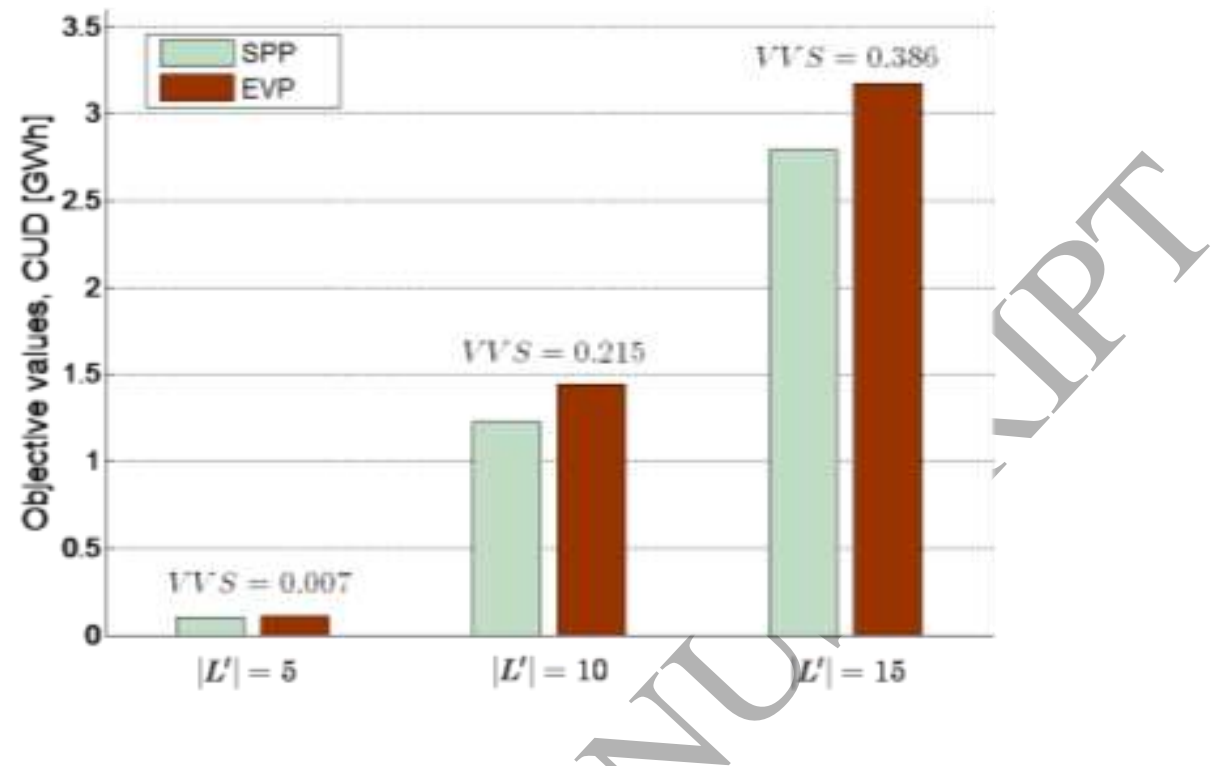

Figure 3. Objectives values and VVSs under different disruption cases

Figure 4 contrasts the restoration curves of the system, i.e. evolution of the system performance level with time, under the five reduced uncertainty scenarios S1-S5 in the SPP against the restoration curve in the EVP for each of the three disruption cases $\left|L^{\prime}\right|=5,10,15$. In Figure 4, the negative part of the $x$-axis (Restoration Period $=-1$ ) indicates a period before the disruption, Restoration Period $=0$ marks the occurrence of the disruption, and the remaining periods time the repair process. The performance level in the $y$-axis represents the percentage of the total demand that can be satisfied. As expected, the loss of system performance increases with the number of damaged transmission line, i.e. $0.37 \%$ for $\left|L^{\prime}\right|=5,3.54 \%$ for $\left|L^{\prime}\right|=10$, and $5.65 \%$ for $\left|L^{\prime}\right|=15$. The small loss of system performance even in the relatively large disruption $\left|L^{\prime}\right|=15$ is the result of redundant transmission lines that compensate for the offline damaged lines. The behavior of small loss of system functionality can be observed more clearly by combining the system restoration curve and the detailed restoration schedule, e.g. the curve of EVP solution in Figure 4(b) (diamond-dotted line) and its Gantt chart in Figure 5. Repairing only two failed lines, i.e. L22 and L81, leads to a full restoration of the system functionality. In terms of the comparison between SPP and EVP solutions, Figure 4 shows that in each disruption case, i.e., $\left|L^{\prime}\right|=5,10,15$, most of the restoration curves under scenarios S1-S5 in SPP 
outperform the restoration curve in EVP (diamond-solid lines), and it is more evident for large disruptions.
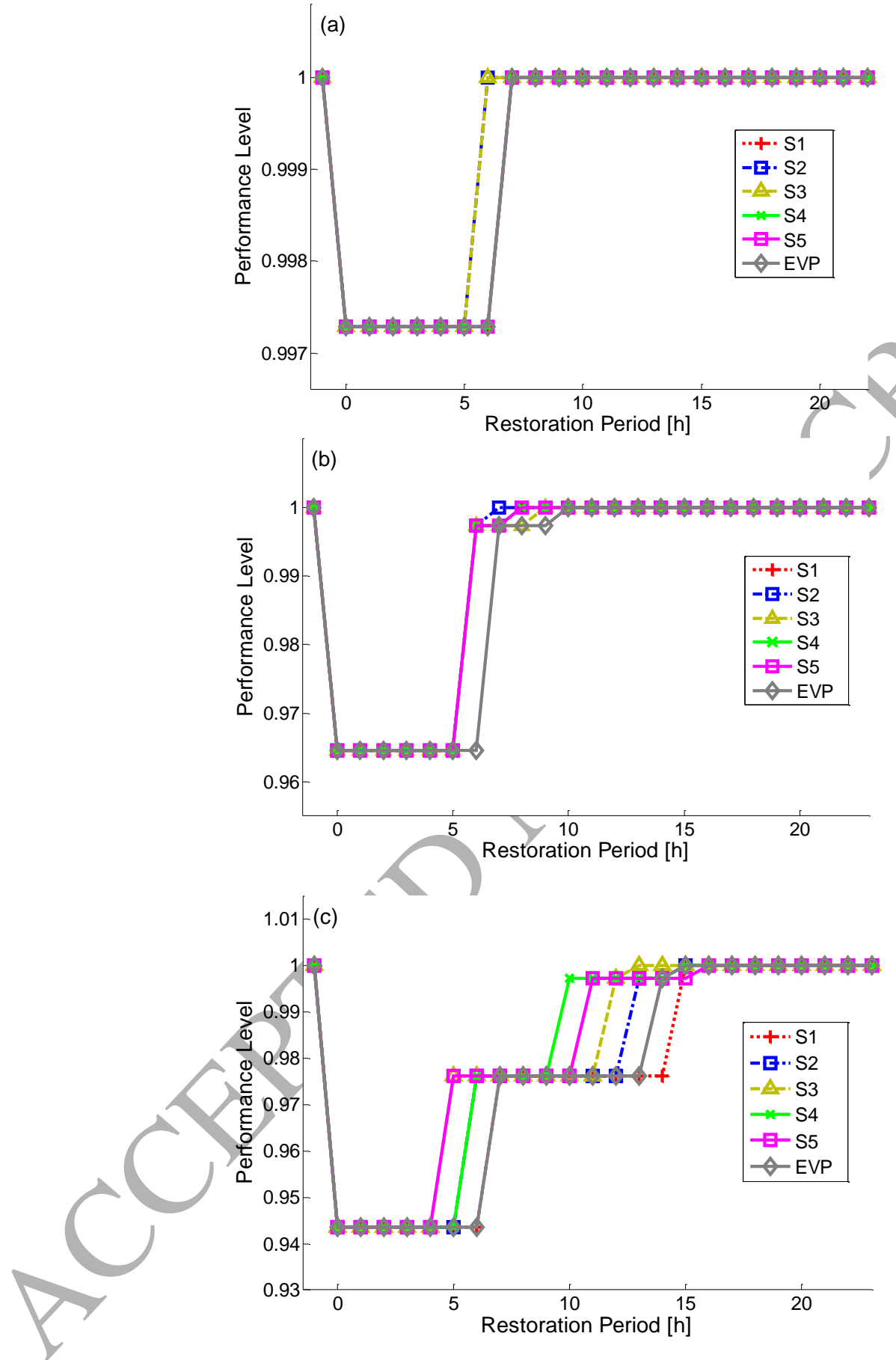

Figure 4. System restoration curves under uncertainty scenarios S1-S5 in SPP and restoration curve in EVP for disruption cases (a) $\left|L^{\prime}\right|=5$, (b) $\left|L^{\prime}\right|=10$, (c) $\left|L^{\prime}\right|=15$. 


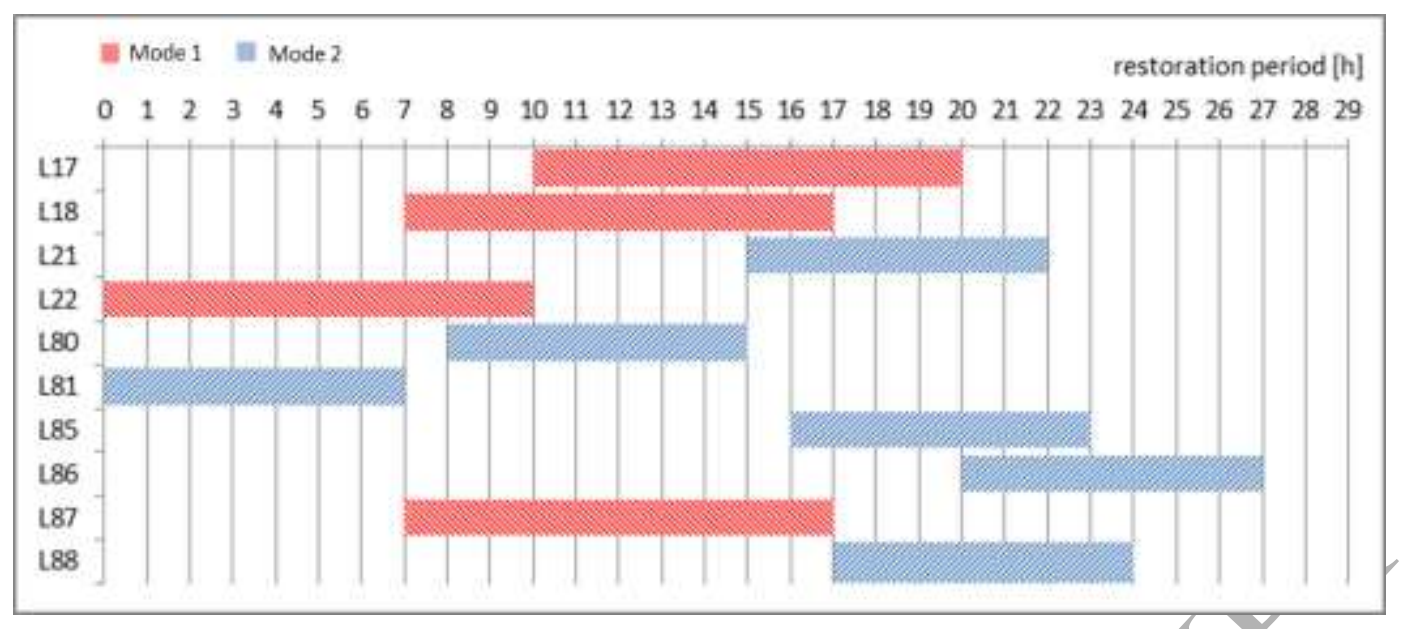

Figure 5. Gantt chart of the detailed restoration process of the EVP solutions for disruption case $\left|L^{\prime}\right|=10$.

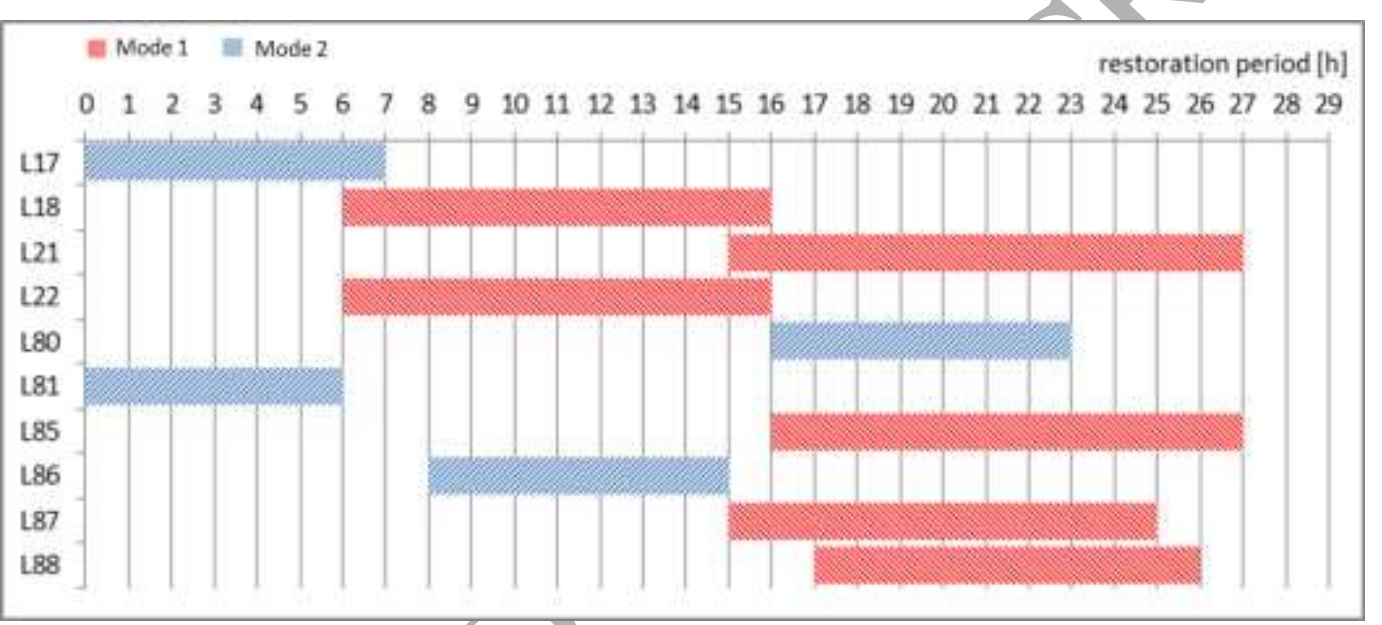

Figure 6. Gantt chart of the detailed restoration process of the SSP solutions for the disruption case $\left|L^{\prime}\right|=10$

and for one of the uncertainty scenarios S2.

Figure 6 shows the Gantt chart of the detailed restoration of the SSP solutions for the uncertainty realization S2 for the disruption case $\left|L^{\prime}\right|=10$. By comparing Figure 6 with Figure 5, one can find that restoration schedules from SSP and EVP are very different. For example, in EVP, lines L22 and L81 are repaired first in mode 1 and 2, respectively, whereas in SSP lines L17 and L81 are repaired first both in mode 2. In other words, consideration of stochasticity in system restoration planning could result in quite different repair modes and time schedule compared to its deterministic counterpart.

Furthermore, we investigate what is the system functionality loss for the solutions of the EVP under each of the five uncertainty scenarios (in terms of the repair duration and available repair crew) other 
than the expected value scenario. Figure 7 shows the CUDs of the EVP solutions under the uncertainty scenarios S1-S5 for the disruption case (a) $\left|L^{\prime}\right|=5$, (b) $\left|L^{\prime}\right|=10$ and (c) $\left|L^{\prime}\right|=15$, and they are compared with the corresponding CUD of the SPP solutions. Figure 7 demonstrates that the SPP solutions result in smaller CUDs than their corresponding EVP solutions in most of the scenarios. Only in four cases, i.e., S2, S5 for $\left|L^{\prime}\right|=5, \mathrm{~S} 3$ for $\left|L^{\prime}\right|=10$ and S1 for $\left|L^{\prime}\right|=15$, the SPP and the EVP solutions entail the same CUDs. These results confirm that applying the EVP solution to each scenario considered in the stochastic problem overestimates the expected objective value CUD, demonstrating the suboptimality of EVP solutions for the CIRPP under uncertainty.
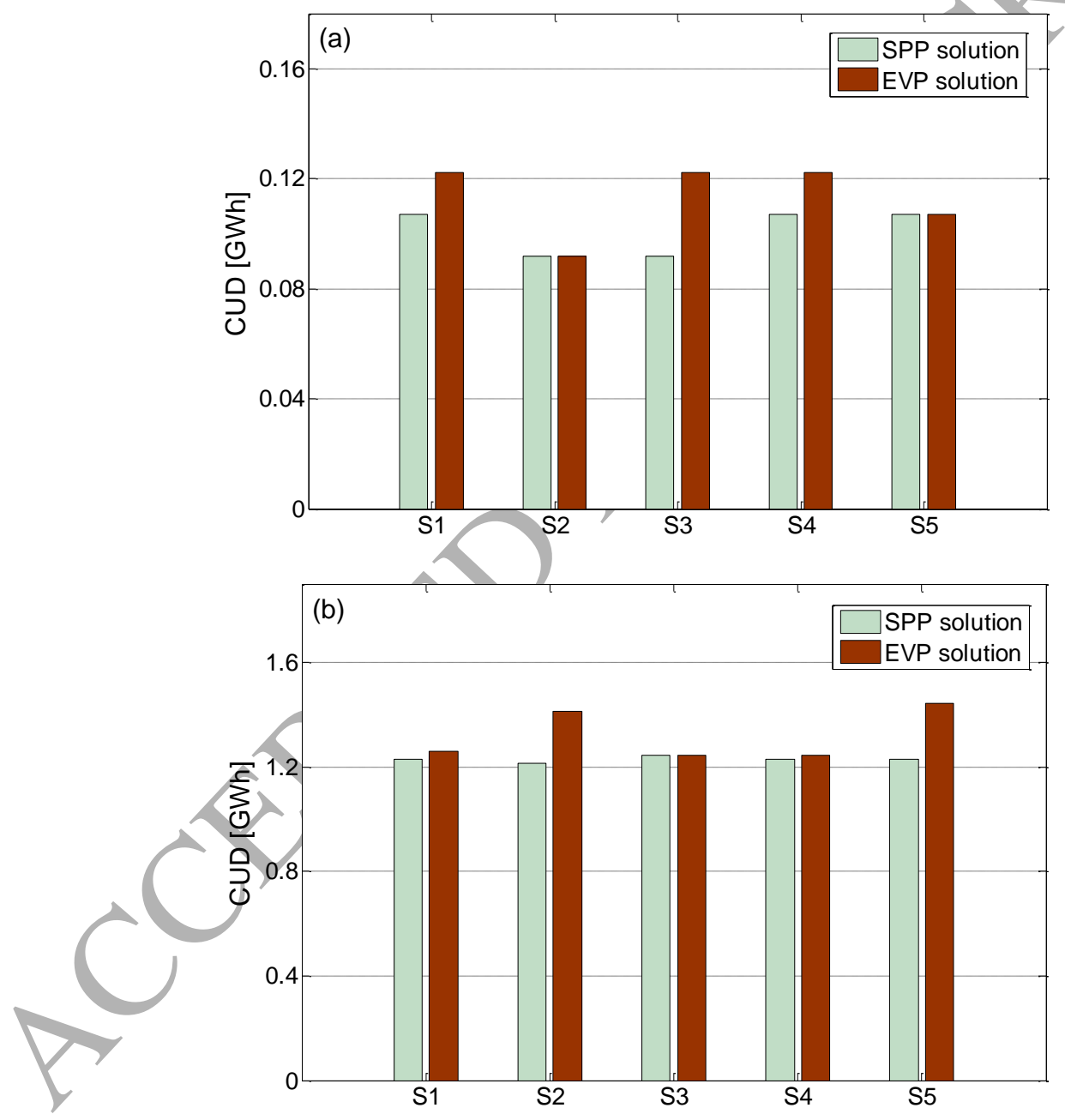


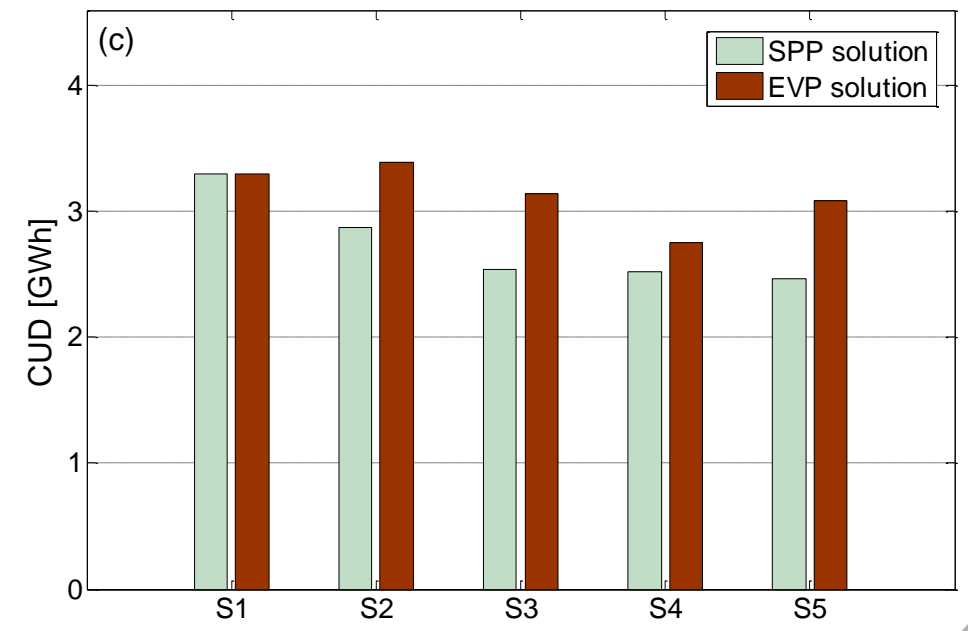

Figure 7. Comparison of CUDs of EVP solutions and SPP solutions under different uncertainty realizations for disruption case (a) $\left|L^{\prime}\right|=5$, (b) $\left|L^{\prime}\right|=10$ and (c) $\left|L^{\prime}\right|=15$.

To study how the solutions of the SPP change under different degrees of uncertainty of the random parameters, Figure 8 shows the CUDs and VSSs of the SPP for increasing variances of the Weibull distribution of the repair durations for the disruption case $\left|L^{\prime}\right|=10$. Larger values of the shape parameter $\alpha$ represent lower variances, and the expected yalues $\beta_{i}^{1} \Gamma\left(1+\alpha_{i}^{-1}\right)=10$ for mode 1 and $\beta_{i}^{2} \Gamma\left(1+\alpha_{i}^{-1}\right)=7$ for mode 2 , are fixed. The EVP/ solution value is the same for all the points in Figure 8 , i.e. $\mathrm{CUD}=1.44 \mathrm{GWh}$. Figure 8 shows that the objective value CUD (to be minimized) of SPP increases as the shape parameter $\alpha$ increases (i.e. the variance decreases), resulting in decreasing VSSs. This is not unexpected because when the variance approaches zero, the VSS is also close to zero, and the random parameters become deterministic. On the other hand, increasing the variance (smaller values of $\alpha$ ) of the Weibull distribution of the repair times results in generally decreases CUDs and increases) VSSs. In other words, as the uncertainty associated with the system restoration planning grows, it is more beneficial to apply the proposed SPP.

Finally, the computational performance of the proposed solution methodology is studied on a machine equipped with $16 \mathrm{~GB}$ of RAM memory and a quad-core $3.6 \mathrm{GHz}$ Intel $17-4790$ processor. The proposed algorithm is implemented using OPL and ILOG script in CPLEX Studio IDE, and CPLEX 12 solvers [50] are used to solve the linear RMP and DSP problems. The results for increasing disruption scenarios, i.e. $\left|L^{\prime}\right|=5,10,15$, are presented in Table 2. Columns 2 and 3 provide information on the problem size, i.e., the number of continuous variables, number of binary variables, 
and number of constraints. Column 4 shows the number of iterations in the Benders decomposition. The total time needed to solve the problem is given in Column 5. The problem size, particularly the number of binary variables and the number of constraints, increases proportionally with the number of damaged lines $\left|L^{\prime}\right|$, resulting in increasing iterations and total computational time. The computational time increases exponentially with the size of the problem, which is a common feature of branch-andcut algorithms, like the one used in this work [50] when addressing MIP problems.

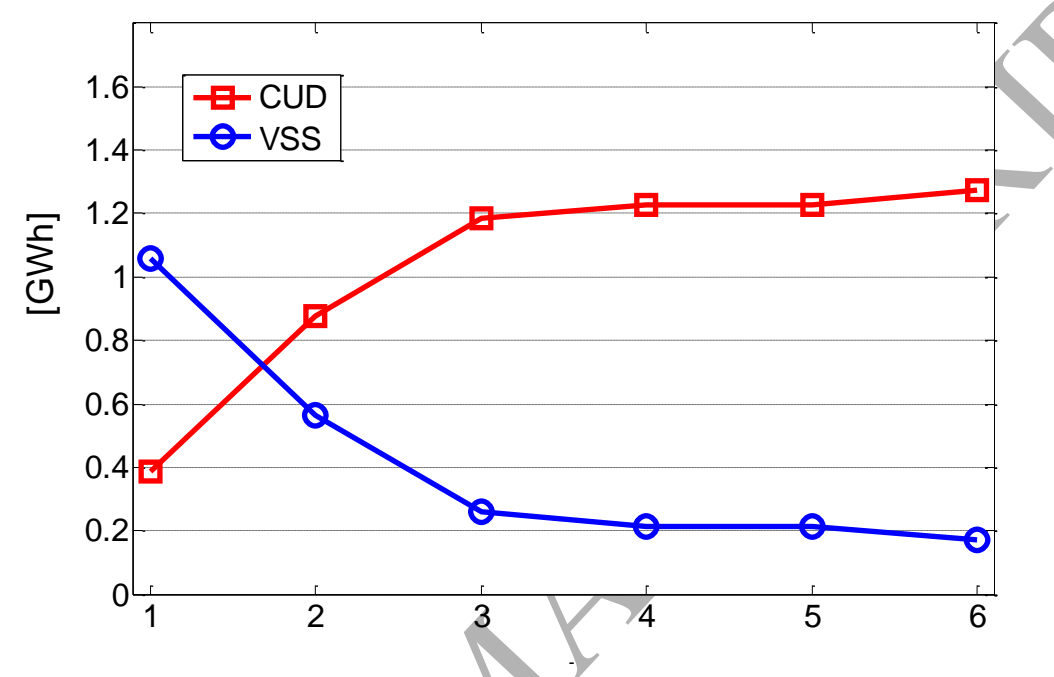

Figure 8. CUDs and VSSs of the SPP under different shape parameters of the Weibull distribution of the repair durations, i.e. different levels of uncertainty, for the disruption case $\left|L^{\prime}\right|=10$.

Table 2. Computational performance of the proposed branch-and-cut algorithm.

\begin{tabular}{cccccc}
\hline \hline \multirow{2}{*}{ Instance } & \multicolumn{3}{c}{ Problem size } & \multirow{2}{*}{ \# iterations } & Computational time (s) \\
\cline { 2 - 4 } & \# cont. variables & \# bin. variables & \# contraints & & \\
\hline$L^{\prime} \mid=5$ & 36331 & 3250 & 27946 & 56 & 175 \\
$\left|L^{\prime}\right|=10$ & 36341 & 6500 & 35251 & 71 & 896 \\
$\left|L^{\prime}\right|=15$ & 36351 & 9750 & 42556 & 103 & 5120 \\
\hline \hline
\end{tabular}

\section{CONCLUSIONS}

This paper proposes a two-stage stochastic programming model for CI restoration planning in which post-disruption restoration activities occur in a highly dynamic environment and are subject to considerable uncertainty. The objective of the model is to minimize the expected loss of performance over all possible realizations of the random parameters, and thus to maximize system resilience. In 
particular, multi-mode restoration activities are introduced, whose tasks can be performed by different allocations of resources and whose duration is, therefore, a function of such allocation. The multimode modeling approach offers more flexibility than its single-mode counterparts, and is more practical for the operators of CI systems. Continuous random variables are sampled using the Latin hypercube technique, and the number of discrete uncertainty scenarios is reduced to a tractable size by applying the fast forward selection algorithm. A tailored Benders decomposition algorithm is applied to solve the resulting mixed-integer equivalence of the original stochastic CIRPP problem.

The proposed approach is applied to a network model based on the British electric power transmission system. The positive values of VSS for the considered disruption scenarios demonstrates the clear benefit of the stochastic method over the deterministic one, which solely employs the expected values of the uncertain parameters. The optimum solutions in terms of repair modes and schedules are largely impacted when uncertainty is accounted for, which further highlights that deterministic restoration processes are suboptimal. Finally, increasing variances of the random parameters in the repair process, i.e., higher levels of uncertainty, results in large values of VSS. These results highlight the need of utilizing a stochastic model for post-disruption restoration planning for critical infrastructure systems, especially when considerable lack of knowledge in decision-making exists.

Two-stage stochastic programming models have always been known as computationally challenging, especially when discrete variables in the second stage problem exist [ $\underline{58}, \underline{59}]$. Hanasusanto et al. [59] shows that linear two-stage stochastic programs with fixed recourse are \#P-hard even if the random problem data is goyerned by independent uniform distributions. The computational time of the proposed algorithm increases exponentially with the problem size and calls for techniques to ensure the applicability of the proposed restoration planning problem to large-scale power systems. To this aim, the study of efficient solution methodologies for two-stage stochastic programming with discrete recourse is an active field of research in mathematical optimization [60]. Scenario reduction techniques, e.g., the fast forward section algorithm used in this paper, have proven to be effective when the original deterministic optimization model is already large scale. Furthermore, disjunctive decomposition [61], can be a promising solution method to test for stochastic CIRPP problems. 
The proposed stochastic programming model for the CIRPP problem presents a flexible framework within which other decision constraints and planning goals can be integrated. For instance, other planning considerations might include ad hoc cost models for the repairing of failed components, the impact of different priorities of demand nodes, and combination of the construction of new components and the repair of failed ones [62]. Moreover, the CIRPP problem in the present study assumes that the selection variables of the repair modes of failed elements are determined at the time of system planning and cannot be altered henceforth. Relaxation of this assumption to adjustable repair mode decisions might be useful in some applications, which makes the problem become a computationally more expensive multi-stage stochastic programming [63]. Investigation in this thread is open to future work. Finally, it would be interesting in our future research to conduct statistical sensitivity analysis of the proposed two-stage stochastic programming (e.g., by adopting so-called response surface methodology [64]) when there is incomplete information about the true probability measures of the uncertain parameters, which may provide decision-makers with valuable information of the obtained optimum solutions with regard to different probability measures of the uncertain parameters (i.e., durations of repair tasks and available resource).

\section{ACKNOWLEDGEMENT}

The authors acknowledge the CTI - Commission for Technology and Innovation (CH), and the SCCER-FURIES - Swiss Competence Center for Energy Research - Future Swiss Electrical Infrastructure, for their financial and technical support to the research activity presented in this paper.

\section{REFERENCES}

[1] Kröger, W. and E. Zio, Vulnerable systems. 2011: Springer Science \& Business Media.

[2] Zio, E., Challenges in the vulnerability and risk analysis of critical infrastructures. Reliability Engineering \& System Safety, 2016. 152: p. 502-512.

[3] Barker, K. and J.R. Santos, A risk-based approach for identifying key economic and infrastructure systems. Risk Analysis, 2010. 30(6): p. 962-974.

[4] Fang, Y.-P. and E. Zio. Optimal production facility allocation for failure resilient critical infrastructures. in ESREL 2013. 2013.

[5] Helbing, D., Globally networked risks and how to respond. Nature, 2013. 497(7447): p. 51-59.

[6] Brown, G., et al., Defending critical infrastructure. Interfaces, 2006. 36(6): p. 530-544. 
[7] Lewis, T.G., Critical infrastructure protection in homeland security: defending a networked nation. 2014: John Wiley \& Sons.

[8] Guikema, S.D., Natural disaster risk analysis for critical infrastructure systems: An approach based on statistical learning theory. Reliability Engineering \& System Safety, 2009. 94(4): p. 855-860.

[9] Park, J., et al., Integrating risk and resilience approaches to catastrophe management in engineering systems. Risk Analysis, 2013. 33(3): p. 356-367.

[10] Fang, Y.P., N. Pedroni, and E. Zio, Resilience-Based Component Importance Measures for Critical Infrastructure Network Systems. IEEE Transactions on Reliability, 2016. PP(99): p. $1-11$.

[11] Dessavre, D.G., J.E. Ramirez-Marquez, and K. Barker, Multidimensional approach to complex system resilience analysis. Reliability Engineering \& System Safety, 2016. 149: p. $34-43$.

[12] Boin, A. and A. McConnell, Preparing for critical infrastructure breakdowns: the limits of crisis management and the need for resilience. Journal of Contingencies and Crisis Management, 2007. 15(1): p. 50-59.

[13] Fang, Y. and G. Sansavini, Optimizing power system investments and resilience against attacks. Reliability Engineering \& System Safety, 2017. 159: p. 161-173.

[14] Hosseini, S., K. Barker, and J.E. Ramirez-Marquez, A review of definitions and measures of system resilience. Reliability Engineering \& System Safety, 2016. 145: p. 47-61.

[15] Vugrin, E.D., M.A. Turnquist, and N.J. Brown, Optimal recovery sequencing for enhanced resilience and service restoration in transportation networks. International Journal of Critical Infrastructures, 2014. 10(3-4): p. 218-246.

[16] Ouyang, M. and Y. Fang, A mathematical framework to optimize critical infrastructure resilience against intentional attacks. Computer-Aided Civil and Infrastructure Engineering, 2017. 32(11): p. 909-929.

[17] Bryson, K.-M.N., et al., Using formal MS/OR modeling to support disaster recovery planning. European Journal of Operational Research, 2002. 141(3): p. 679-688.

[18] Casari, M. and S.J. Wilkie, Sequencing lifeline repairs after an earthquake: an economic approach. Journal of Regulatory Economics, 2005. 27(1): p. 47-65.

[19] Lee, E.E., J.E. Mitchell, and W.A. Wallace, Restoration of services in interdependent infrastructure systems: A network flows approach. Systems, Man, and Cybernetics, Part C: Applications and Reviews, IEEE Transactions on, 2007. 37(6): p. 1303-1317.

[20] Matisziw, T.C., A.T. Murray, and T.H. Grubesic, Strategic network restoration. Networks and Spatial Economics, 2010. 10(3): p. 345-361. 
[21] Nurre, S.G., et al., Restoring infrastructure systems: An integrated network design and scheduling (INDS) problem. European Journal of Operational Research, 2012. 223(3): p. 794806.

[22] Nurre, S.G. and T.C. Sharkey, Integrated network design and scheduling problems with parallel identical machines: Complexity results and dispatching rules. Networks, 2014. 63(4): p. 306-326.

[23] González, A.D., et al., The Interdependent Network Design Problem for Optimal Infrastructure System Restoration. Computer-Aided Civil and Infrastructure Engineering, 2015.

[24] Ouyang, M. and Z. Wang, Resilience assessment of interdependent infrastructure systems: With a focus on joint restoration modeling and analysis. Reliability Engineering \& System Safety, 2015. 141: p. 74-82.

[25] Klerides, E. and E. Hadjiconstantinou, A decomposition-based stochastic programming approach for the project scheduling problem under timelcost trade-off settings and uncertain durations. Computers \& Operations Research, 2010. 37(12):p. 2131-2140.

[26] Van Hentenryck, P. Computational Disaster Management. in IJCAI. 2013.

[27] Xu, N., et al., Optimizing scheduling of post-earthquake electric power restoration tasks. Earthquake engineering \& structural dynamics, 2007. 36(2): p. 265-284.

[28] Herroelen, W. and R. Leus, Project scheduling under uncertainty: Survey and research potentials. European journal of operational research, 2005. 165(2): p. 289-306.

[29] Hartmann, S. and D. Briskorn, A survey of variants and extensions of the resourceconstrained project scheduling problem. European Journal of operational research, 2010. 207(1): p. 1-14.

[30] Cardin, M.-A., et al., An Approach for Analyzing and Managing Flexibility in Engineering Systems Design Based on Decision Rules and Multistage Stochastic Programming. IISE Transactions, 2016,49(1): p. 1-12.

[31] Birge, J.R. and F. Louveaux, Introduction to stochastic programming. 2011: Springer Science \& Business Media.

[32] Conejo, A.J., M. Carrión, and J.M. Morales, Decision making under uncertainty in electricity markets. Vol. 1. 2010: Springer.

[33] Albright, S.C., Optimal maintenance-repair policies for the machine repair problem. Naval Research Logistics (NRL), 1980. 27(1): p. 17-27.

[34] Marseguerra, M., E. Zio, and L. Podofillini, Condition-based maintenance optimization by means of genetic algorithms and Monte Carlo simulation. Reliability Engineering \& System Safety, 2002. 77(2): p. 151-165. 
[35] Marseguerra, M. and E. Zio, Optimizing maintenance and repair policies via a combination of genetic algorithms and Monte Carlo simulation. Reliability Engineering \& System Safety, 2000. 68(1): p. 69-83.

[36] Wang, W., A joint spare part and maintenance inspection optimisation model using the delaytime concept. Reliability Engineering \& System Safety, 2011. 96(11): p. 1535-1541.

[37] Pandey, M., et al., Selective maintenance for binary systems under imperfect repair. Reliability Engineering \& System Safety, 2013. 113: p. 42-51.

[38] Ahuja, R.K., T.L. Magnanti, and J.B. Orlin, Network flows: theory, algorithms, and applications. 1993: Prentice Hall.

[39] Fang, Y. and G. Sansavini. Optimum post-disruption restoration for enhánced infrastructure network resilience: a fuzzy programming approach. in ESREL 2016. 2016.

[40] Abdelkader, Y.H., Evaluating project completion times when activity times are Weibull distributed. European Journal of Operational Research, 2004. 157(3): p. 704-715.

[41] Ahmed, S., Two-Stage Stochastic Integer Programming: A Brief Introduction. Wiley Encyclopedia of Operations Research and Management Science.

[42] Crainic, T.G., Y. Li, and M. Toulouse, A first multilevel cooperative algorithm for capacitated multicommodity network design. Computers \& Operations Research, 2006. 33(9): p. $2602-$ 2622.

[43] Yi, W. and A. Kumar, Ant colony optimization for disaster relief operations. Transportation Research Part E: Logistics and Transportation Review, 2007. 43(6): p. 660-672.

[44] Wyss, G.D. and K.H. Jorgensen, A user's guide to LHS: Sandia's Latin hypercube sampling software. 1998.

[45] Dupačová, J., N. Gröwe-Kuska, and W. Römisch, Scenario reduction in stochastic programming. Mathematical programming, 2003. 95(3): p. 493-511.

[46] Heitsch, H. and W. Römisch, Scenario reduction algorithms in stochastic programming. Computational optimization and applications, 2003. 24(2-3): p. 187-206.

[47] Morales, J.M., et al., Scenario reduction for futures market trading in electricity markets. Power Systems, IEEE Transactions on, 2009. 24(2): p. 878-888.

[48] Geoffrion, A.M., Generalized benders decomposition. Journal of optimization theory and applications, 1972. 10(4): p. 237-260.

[49] Floudas, C.A., Nonlinear and mixed-integer optimization: fundamentals and applications. 1995: Oxford University Press.

[50] CPLEX, I.I., V12. 1: Users manual for CPLEX. International Business Machines Corporation, 2009. 46(53): p. 157.

[51] Bell, K.W. Test system requirements for modelling future power systems. in IEEE PES General Meeting. 2010. IEEE. 
[52] Bukhsh, W. and K. McKinnon, Network data of real transmission networks. Published online at http://www.maths.ed.ac.uk/optenergy/NetworkData, 2013.

[53] Force, R.T., The IEEE reliability test system-1996. IEEE Trans. Power Syst, 1999. 14(3): p. 1010-1020.

[54] Wahlquist, C., South Australia storms: entire state left without power after wild weather - as it happened, in theguardian. 2016, theguardian.

[55] Çagnan, Z. and R. Davidson. Post-earthquake restoration modeling of electric power systems. in the 13th World Conference on Earthquake Engineering. 2004.

[56] Birge, J.R., The value of the stochastic solution in stochastic linear programs with fixed recourse. Mathematical programming, 1982. 24(1): p. 314-325.

[57] DECC, UK Energy Statistics, 2015 \& Q4 2015. 2016, Department of Energy and Climate Change. p. 2.

[58] Dyer, M. and L. Stougie, Computational complexity of stochastic programming problems. Mathematical Programming, 2006. 106(3): p. 423-432.

[59] Hanasusanto, G.A., D. Kuhn, and W. Wiesemann, A comment on "computational complexity of stochastic programming problems". Mathematical Programming, 2016. 159(1-2): p. 557569.

[60] Ahmed, S., Two-Stage Stochastic Integen Programming: A Brief Introduction. Wiley Encyclopedia of Operations Research and Management Science, 2010.

[61] Ntaimo, L., Disjunctive decomposition for two-stage stochastic mixed-binary programs with random recourse. Operations research, 2010. 58(1): p. 229-243.

[62] Fang, Y. and G. Sansavini, Emergence of Antifragility by Optimum Postdisruption Restoration Planning of Infrastructure Networks. Journal of Infrastructure Systems, 2017. 23(4): p. 04017024.

[63] Shapiro, A., D. Dentcheva, and A. Ruszczyński, Lectures on stochastic programming: modeling and theory. 2009: SIAM.

[64] Bailey, T.G., P.A. Jensen, and D.P. Morton, Response surface analysis of two-stage stochastic linear programming with recourse. Naval Research Logistics, 1999. 46(7): p. 753-776. 